\title{
I SISTEMI ELETTORALI MISTI. UNA CLASSIFICAZIONE
}

\author{
di Alessandro Chiaramonte
}

Le riforme elettorali negli anni' '90

A partire dagli anni '20-30, epoca dell'introduzione della proporzionale in molti paesi e dell'avvio del processo di strutturazione della politica di massa (Rokkan 1970), e fino all'inizio di questo decennio, i sistemi elettorali dei regimi democratici hanno mostrato una straordinaria continuità.

Sebbene più volte siano state attuate riforme di certi meccanismi concernenti, ad esempio, la soglia legale di accesso alla rappresentanza, la dimensione dei collegi e il loro disegno, ed anche la formula, si deve tuttavia rilevare che esse hanno avuto luogo pressoché esclusivamente entro i limiti del rispettivo principio di rappresentanza (proporzionale/maggioritario), eccettuati i casi di discontinuità di regime (Nohlen 1984b). Come dire che i sistemi elettorali sono stati sì talvolta ritoccati, ma non così il loro impianto fondamentale - se non in circostanze storiche ben definite (come nel passaggio dalla IV alla V Repubblica in Francia) ${ }^{1}$.

Desidero ringraziare Leonardo Morlino e Roberto D'Alimonte, nonché $i$ due anonimi referees, per $i$ preziosi suggerimenti che mi banno offerto. Va da sé che io sia il solo responsabile del contenuto del saggio e dei suoi eventuali errori.

1 Sono state formulate varie ipotesi per spiegare tale continuità. Reeve e Ware (1992), impiegando un approccio razionalistico, citano motivazioni quali l'incapacità di prevedere gli effetti dei meccanismi elettorali, la presenza di vincoli costituzionali, la circostanza che i governanti siano i beneficiari del funzionamento di un sistema elettorale, la difficoltà di modificare le regole del gioco senza il consenso dell'opinione pubblica o di un accordo con l'opposizione (pp. 10-14). In ultima analisi, tuttavia, la ragione principale sembra essere stata quella per cui i sistemi elettorali - che fossero costituzionalizzati o meno - e più ancora il principio di rappresentanza da essi incarnato sono stati percepiti come una legge fondamentale, facente parte di quell'accordo-compromesso e di quel sistema dei valori alla base del regime politico stesso (Nohlen 1984b).

RIVISTA ITALIANA DI SCIENZA POLITICA / a. XXVIII, n. 2, agosto 1998 
Negli anni '90, però, la continuità dei sistemi elettorali quanto al principio di rappresentanza è venuta meno in più di una occasione anche in regimi democratici di antico consolidamento che non necessariamente hanno sperimentato (o completato) una vera transizione di regime. Ciò si è verificato soprattutto in seguito ad un processo di destrutturazione partitica testimoniato dalla nascita di nuove formazioni politiche e dall'aumento dei livelli di volatilità elettorale - che ha minato le basi stesse della struttura istituzionale in cui quei sistemi elettorali sono emersi. L'Italia, la Nuova Zelanda e il Giappone costituiscono un chiaro esempio di paesi che, sotto l'urto di vicende interne, hanno profondamente cambiato i loro meccanismi di elezione $^{2}$. Anche più interessante è il fatto che tali paesi, seguendo un percorso molto diverso se non opposto, hanno finito tutti per adottare sistemi elettorali che combinano allo stesso tempo elementi tipici sia dei sistemi maggioritari che dei sistemi proporzionali. Hanno cioè optato per sistemi elettorali che, possibilmente, coniugassero i vantaggi di ciascuno dei due principi di rappresentanza e attenuassero anche i relativi svantaggi.

Italia, Nuova Zelanda e Giappone non sono peraltro gli unici casi nei quali la riforma dei sistemi elettorali nazionali ha portato all'introduzione di una formula mista. La transizione alla democrazia che ha avuto avvio nei paesi ex-comunisti dell'Europa orientale a partire dalla fine degli anni ' 80 e che nello stesso periodo ha compiuto qualche ulteriore passo in alcuni paesi dell'America latina e dell'Asia si è accompagnata, a seconda dei casi, ad una ricostruzione o ad una correzione dei meccanismi istituzionali fondamentali, compresa la legge elettorale. E il risultato è che Ungheria, Bulgaria, Albania, Croazia, Lituania, Russia, Georgia e ancora Messico, Venezuela, Corea del Sud e Taiwan hanno anch'essi, come i tre paesi prima citati, optato per un sistema elettorale misto maggioritario-proporzionale. Come si può ben vedere, la Germania, che a lungo è rimasto l'unico esempio di applicazione di questo tipo di sistemi elettorali - dopo che solo la Danimarca nelle elezioni del 1918 e la

$2 \grave{E}$ ancora presto comunque per affermare che l'introduzione dei nuovi sistemi elettorali in questi paesi non rientri in una sequenza di discontinuità di regime. In Italia è infatti in corso un processo di revisione costituzionale il cui approdo è ancora da valutare. Per quanto poi riguarda la Nuova Zelanda, la Commissione reale che ha proposto l'adozione del nuovo sistema elettorale, poi avvenuta tramite referendum popolare, lo ha fatto anche in vista del passaggio ad un modello consensuale di democrazia (Lijphart 1987; Boston 1987). 
Francia nel periodo 1951-56 avevano sperimentato una combinazione di elementi maggioritari e proporzionali -, si trova adesso in compagnia di molti altri paesi.

Detto che molte riforme elettorali di questi ultimi dieci anni hanno avuto un comune denominatore nell'adozione di sistemi elettorali misti, si tratta ora di chiarire cosa siano effettivamente quest'ultimi e, ancor prima, se, e in che senso, costituiscano un tertium genus rispetto ai sistemi maggioritari e a quelli proporzionali - il che, a sua volta, investe il problema della classificazione dei sistemi elettorali. Questi argomenti sono l'oggetto delle due sezioni che seguono.

Maggioritario e proporzionale: ovvero della classificazione dei sistemi elettorali

Il procedimento di classificazione dei sistemi elettorali dà origine a una serie complessa di problemi: la ricerca del giusto equilibrio tra parsimonia e capacità discriminante delle classi individuate, quale sia il criterio (o i criteri) di classificazione più appropriato, se le classi siano davvero esaustive rispetto all'universo di riferimento, se la distinzione tra i sistemi elettorali sia una questione di gradi o di qualità. Si tratta di problemi ai quali sono state fornite soluzioni molto diverse che occorre qui ripercorrere brevemente, in modo da comprendere il senso di concetti quali maggioritario e proporzionale e, quindi, - cosa che più interessa in questa sede - cosa si intende per sistemi elettorali misti maggioritario-proporzionali.

Il criterio più comunemente impiegato per classificare i sistemi elettorali è il tipo di formula elettorale adottata, ossia la regola aritmetica di conversione dei voti in seggi. Su questa base i sistemi elettorali si dicono maggioritari se utilizzano le formule plurality (a maggioranza relativa) o majority (a maggioranza assoluta), mentre si qualificano come proporzionali se utilizzano quelle d'Hondt, Saint-Laguë, Hare, ecc. Questa classificazione ha certamente il vantaggio della semplicità, ma, viene obbiettato, da un lato sacrifica importanti informazioni - la formula è infatti soltanto una, e neanche la più importante, delle dimensioni descrittive dei sistemi elettorali (Rae 1967; Taagepera e Shugart 1989) -, dall'altro è imperniata esclusivamente sugli input legali (il «come è» del sistema elettorale) e quindi trascura gli output elettorali (il «cosa fa» del sistema elettorale). 
Il riconoscimento che la formula non è il solo elemento significativo di un sistema elettorale conduce alla costruzione più o meno esplicita - di tipologie dei sistemi elettorali che tengano conto di tutte le loro dimensioni costitutive, quindi anche dell'ampiezza dei collegi, delle soglie di accesso alla rappresentanza, ecc. Queste tipologie hanno indubbiamente il pregio di offrire una vasta gamma di informazioni, nonché di favorire la selezione dei fattori rilevanti (le variabili indipendenti) per l'analisi degli effetti dei sistemi elettorali. Tuttavia, la loro complessità va talvolta a scapito della parsimonia. Non a caso anche coloro che hanno contribuito ad affinarle poi difficilmente sfuggono al ricorso di termini quali maggioritario e proporzionale con riferimento, ancora una volta, alla formula - quando si tratta di distinguere tra tipi principali di sistemi elettorali (si veda, ad esempio, Blais 1988 e 1991).

Lo spostamento del focus analitico dagli input agli output dà luogo ad una classificazione basata sugli effetti prodotti dai sistemi elettorali, in particolare sul sistema partitico. Duverger (1951) opera una distinzione di nuovo tra sistemi maggioritari, quelli che tendono a produrre un formato bipartitico, e sistemi proporzionali, quelli che tendono a produrre un formato multipartitico. È da notare però che una tale classificazione non genera classi empiriche identiche a quella che discende dal tipo di formula adottata, poiché i sistemi con formula majority (ad esempio la Francia) sono associati al multipartitismo, ragion per cui, sempre secondo Duverger, devono essere ritenuti analoghi ai sistemi proporzionali. Anche la classificazione dei sistemi elettorali di Duverger, però, non rimane immune da critiche. In special modo si rileva che la capacità discriminante delle classi individuate risulta limitata, poiché formati identici del sistema di partito sono associati a sistemi elettorali congegnati in modo tra loro molto differente ${ }^{3}$.

La linea argomentativa di Duverger e, quindi, la classificazione dalla prospettiva dell'output, viene riproposta con alcune correzioni da altri autori. Ne consegue un approfondimento del nesso esistente tra i sistemi elettorali e il numero di partiti e, soprattutto, tra i primi e il grado di disproporzionalità prodotta.

3 Due esempi classici sono il Canada e l'Austria. Il primo configura il caso di un sistema multipartitico pur in presenza di un sistema plurality, il secondo è stato a lungo un sistema bipartitico nonostante l'impiego di un sistema elettorale proporzionale. Per una critica a Duverger si rimanda a Sartori (1984). 
Utilizzando concetti che si prestano facilmente ad una quantificazione e si traducono in variabili operative di tipo continuo quali il numero di partiti e la disproporzionalità - , la differenza tra i sistemi elettorali finisce per diventare così una questione di gradi e non di qualità. È esemplificativa al riguardo la posizione di Rose (1984), che, sulla base dell'applicazione di un indice di disproporzionalità, rileva come le differenze tra i sistemi maggioritari e quelli proporzionali siano in realtà minime. Seguendo questa impostazione, in sostanza, la distinzione tra sistemi maggioritari e sistemi proporzionali sfuma: maggioritario diviene sinonimo di non o poco proporzionale.

Diversamente da Rose, Nohlen (1984a) salva ed anzi sottolinea l'importanza dei concetti di maggioritario e di proporzionale servendosene per qualificare quelli che definisce come due principi di rappresentanza tra loro incompatibili. Nohlen sposta il fuoco del problema dall'aspetto descrittivo-interpretativo all'aspetto normativo. L'idea del principio di rappresentanza è storicamente antecedente a quella dei sistemi elettorali. Ma di quest'ultimi essa ha poi determinato gli obbiettivi. Il rapporto che esiste tra sistemi elettorali e principio di rappresentanza è, infatti, proprio un rapporto mezzi-fini. La distinzione tra maggioritario e proporzionale assume allora un valore nella definizione degli obbiettivi propri a ciascuno dei due principi di rappresentanza, vale a dire:

1) il principio maggioritario di rappresentanza ha come suo obbiettivo quello di consentire ad un partito il conseguimento della maggioranza parlamentare - anche laddove esso non abbia una maggioranza di voti - onde produrre un governo (Nohlen 1984a, 86-87);

2) il principio proporzionale di rappresentanza ha come suo obbiettivo quello di far sì che l'assemblea parlamentare rifletta quanto più possibile la composizione delle forze sociali e dei gruppi politici della popolazione (Nohlen 1984a, 87).

In questo senso la dicotomia maggioritario/proporzionale evoca quelle di responsabilità (accountability)/rappresentatività e di stabilità di governo/ricettività del parlamento (Blais 1991; Sartori 1968), e serve per valutare il grado al quale i sistemi elettorali approssimano gli obbiettivi del principio di rappresentanza che si suppone incorporino. Maggioritario e proporzionale sarebbero dunque categorie a priori, ossia elementi normativi che fanno parte di quell'accordo-compromesso che è alla base di un regime politico democratico e del quale contribuiscono a 
definire gli obbiettivi (sebbene non sia detto che riescano a soddisfarli).

Da un'altra prospettiva, però, le tesi di Nohlen e di Rose non sono necessariamente antinomiche. La prima insiste su una distinzione che assurge a parametro di riferimento in sede di valutazione del rendimento dei sistemi elettorali, rendimento che - come detto - si raccorda con il principio di rappresentanza da essi incorporato. Accettando la distinzione tra maggioritario e proporzionale, cioè, non è più legittimo valutare i sistemi elettorali fondati su un principio di rappresentanza mediante i criteri normativi derivanti dall'altro. La seconda, la tesi di Rose secondo cui la differenza tra sistemi elettorali è una questione di gradi, si applica invece propriamente in sede di analisi, laddove la rilevazione comparata degli effetti da essi esercitati deve compiersi uniformemente, ossia utilizzando le stesse variabili e, se possibile, gli stessi indici.

Per ricapitolare, $\mathrm{i}$ termini maggioritario e proporzionale sono stati impiegati per indicare, di volta in volta o anche simultaneamente: 1) il tipo di formule elettorali adottate; 2) il tipo di effetti esercitati sul sistema partitico; 3) gli estremi del continuum dei possibili livelli di (dis)proporzionalità prodotti nella conversione dei voti in seggi; 4) un principio di rappresentanza. Se ne dovrebbe concludere che essi siano concetti svaporati, privi ormai, a causa della loro vaghezza, di una qualsiasi capacità discriminante. Eppure, sembra impossibile fare a meno del loro potere evocativo. Maggioritario e proporzionale, infatti, non sono solo nozioni a tutt'oggi utilizzate dagli studiosi che si occupano di sistemi elettorali; esse sono diffuse e conosciute anche presso un pubblico più vasto. Il punto decisivo riguarda allora il cosa al quale essi sono riferiti. Non si può cioè eludere la specificazione del significato con il quale li si impiega, tanto più se - come in questo caso - si ha a che fare con sistemi elettorali che si definiscono in base alla loro combinazione.

\footnotetext{
4 Rose però compie un altro tipo di errore. Quando afferma che le differenze tra sistemi maggioritari e sistemi proporzionali sono minime trae dai valori del suo indice di disproporzionalità più informazioni di quante essi in realtà diano. La disproporzionalità, infatti, riguarda la sola fase di conversione dei voti in seggi e sconta perciò gli effetti che si sono esplicati in una fase precedente, ossia nella trasformazione delle preferenze in voti - ove i sistemi maggioritari hanno un impatto ben più forte di quelli proporzionali.
} 
Sistemi elettorali misti: una definizione

La definizione di sistema elettorale misto che viene qui adottata discende dalla classificazione dei sistemi elettorali che insiste sugli aspetti descrittivi (gli input) più che sul tipo di effetti prodotti (gli output). E tuttavia essa non rinuncia a evocare anche una componente normativa.

Dal punto di vista puramente descrittivo, diremo allora che i sistemi elettorali misti sono quei sistemi che impiegano (almeno) due differenti formule - l'una maggioritaria e l'altra proporzionale - simultaneamente in una stessa elezione, a patto che i rappresentanti eletti grazie a una delle due formule siano almeno il 5\% di quelli eletti grazie all'altra (Blais e Massicotte 1996) $)^{5}$. La componente normativa che fa da corollario a questa definizione risiede nell'assunzione che i sistemi elettorali misti impiegano due formule diverse per l'assegnazione dei seggi nel tentativo di compendiare i principi maggioritario e proporzionale di rappresentanza, ovverosia due logiche distinte di competizione tra i partiti e di selezione del personale politico-parlamentare.

Occorrono un paio di precisazioni. Innanzitutto, è bene ribadire il significato con il quale si fa uso qui dei termini maggioritario e proporzionale. Essi stanno ad indicare, descrittivamente, una formula elettorale e, normativamente, un principio di rappresentanza. La dizione di sistema elettorale misto, perciò, non presuppone necessariamente effetti distorsivi e selettivi mediani rispetto ai sistemi con sola formula maggioritaria e ai sistemi con sola formula proporzionale. I sistemi misti possono rivelarsi cioè più proporzionalistici dei sistemi proporzionali o più disproporzionalistici dei sistemi maggioritari senza che ciò pregiudichi la loro natura composita e il loro trattamento di categoria a sé stante.

Quando, poi, si assume che i sistemi misti incorporano, combinandoli, i principi maggioritario e proporzionale di rappresentanza, lo si fa a prescindere dalla considerazione che, come accaduto palesemente in molti paesi dell'Europa dell'Est, essi siano stati il frutto di una soluzione di compromesso tra la

5 Sulla base di questa definizione, il significato di sistema misto differisce qui da quello attribuitogli da Lijphart (1984), il quale preferisce riservare tale denominazione per sistemi plurality che contemplano norme per la rappresentazione delle minoranze (come il vecchio sistema neozelandese con i Maori). 
vecchia élite e le forze protagoniste della democratizzazione piuttosto che di una scelta corrispondente ad una precisa volontà di mediare $\mathrm{i}$ due principi di rappresentanza (Kasapovic 1995). Il punto è che essi sono il prodotto di due logiche storicamente contrapposte di cui provano a sommare i rispettivi elementi di forza. Detto in altro modo, i sistemi elettorali misti aspirano a coniugare «il meglio dei due mondi» (Lijphart 1984; Elklit 1992) - del «mondo» maggioritario così come del «mondo» proporzionale.

Per specificare più in concreto il significato attribuito ai sistemi elettorali misti e anche per fugare eventuali equivoci circa i casi empirici che rientrano in tale categoria, è bene anticipare sin d'ora che qui consideriamo il sistema elettorale tedesco come un sistema misto a pieno titolo. Questa scelta, peraltro consequenziale alla definizione appena formulata, potrebbe apparire ad alcuni del tutto inappropriata, in virtù del fatto che, come noto, il sistema tedesco produce esiti sostanzialmente proporzionalistici, attenuati solo dall'impiego di una clausola di sbarramento piuttosto elevata $(5 \%)$ e comunque non dall'assegnazione della metà dei seggi in collegi uninominali mediante formula plurality ${ }^{6}$. Tuttavia, non sfugge a nessuno che l'inclusione di questo sistema elettorale nella classe dei sistemi proporzionali ha sempre creato un qualche imbarazzo. Ne è prova la proliferazione delle etichette utilizzate per riassumerne la peculiare natura data dalla compresenza di una formula maggioritaria e di una formula proporzionale: tra le altre, sistema «di proporzionale personalizzata» (Nohlen 1978; Kaase 1984), «a membro aggiuntivo» (Irvine 1984), «a due voti» (Jesse 1995), «a seggio compensativo» (Taagepera e Shugart 1989), «proporzionale a membro misto» (Boston 1987) ${ }^{7}$. In realtà il sistema elettorale tedesco incontra perfettamente la nostra definizione di sistema misto, che, per ripetere, guarda ad esso per «come è» e non per «cosa fa». Del resto non si capirebbe perché esso, che è certamente «misto» «in entrata», debba essere considerato proporzionale per via degli effetti prodotti sul sistema partitico al pari di altri sistemi elettorali - si pensi, ad esempio, a quelli vigenti in Spagna e in Grecia - che adottano sì una for-

6 Per una descrizione più puntuale del funzionamento del sistema tedesco si rimanda alla sezione dedicata ai sistemi misti a correzione.

7 Il sistema tedesco è stato poi definito più volte «misto», ma con un'accezione non del tutto coincidente con quella attribuita in questa sede. 
mula solo proporzionale ma che producono esiti molto più disproporzionalistici, addirittura comparabili a quelli dei sistemi maggioritari. Ma questa non è l'unica ragione che legittima l'appartenenza del sistema tedesco alla classe dei sistemi elettorali misti. Tanto la sua origine storica (il cosiddetto metodo Geyerhahn) quanto la sua logica composita di competizione e di voto, infatti, lo caratterizzano inequivocabilmente come un sistema che combina elementi tipici dei sistemi maggioritari e dei sistemi proporzionali.

\section{Varietà di sistemi misti}

Fatta luce sul significato che deve attribuirsi al concetto di sistema elettorale misto e giustificato per esso l'utilizzo di una categoria analitica autonoma, si tratta di individuare differenze e similarità dei casi empirici che rientrano all'interno di detta categoria. In altre parole, è necessario a questo punto scomporre la classe dei sistemi elettorali misti in sotto-gruppi più omogenei. A tal fine occorre stabilire $\mathrm{i}$ criteri più idonei a farci comprendere come essi siano diversamente congegnati. Si potrebbe immediatamente pensare che la quota relativa di seggi assegnati mediante formula maggioritaria (o proporzionale) sia al proposito l'elemento cruciale; $o$, in alternativa, che lo sia la soglia effettiva (effective threshold), vale a dire il potenziale di (dis)proporzionalità determinato dalla combinazione dell'entità della soglia legale di accesso alla rappresentanza e della dimensione media dei collegi. Come ampiamente dimostrato dalla letteratura sui sistemi elettorali, sono infatti questi i fattori fondamentali che spiegano il diverso impatto sui sistemi di partito, fatte salve le peculiarità di ciascun contesto di applicazione. Tuttavia, nel caso dei sistemi elettorali misti procedere ad una loro classificazione a partire da queste variabili sarebbe fuorviante. Non che esse non contino - conservano comunque una grande importanza - ma, come risulterà più chiaro tra breve, ai fini della classificazione dei sistemi misti è preliminare ad ogni altra considerazione il modo in cui il livello maggioritario e il livello proporzionale di ripartizione dei seggi sono raccordati.

Secondo Blais e Massicotte (1996) le differenze nel raccordo proporzionale-maggioritario sono riconducibili a due variabili: 1) la separazione o fusione delle procedure di ripartizione dei seggi, con la quale si intende che l'applicazione delle due for- 
mule avviene in modo reciprocamente indipendente ovvero dipendente; 2) la separazione o sovrapposizione delle aree territoriali in cui operano la formula maggioritaria e quella proporzionale. Chiameremo l'una separazione meccanica e l'altra separazione territoriale.

Sulla base di queste variabili Blais e Massicotte individuano tre differenti modalità di connessione tra i livelli maggioritario e proporzionale di ripartizione dei seggi, alle quali corrispondono altrettanti tipi di sistemi elettorali misti: la coesistenza (quando vi è separazione meccanica e territoriale), la combinazione (quando vi è separazione meccanica ma non territoriale) e la correzione (quando non vi è né separazione meccanica né territoriale, ovvero quando i due livelli sono assolutamente interdipendenti).

Rispetto alla classificazione appena illustrata occorre una precisazione. Come sottolineato dagli stessi Blais e Massicotte, in uno stesso sistema elettorale misto è possibile rinvenire contemporaneamente più modalità di raccordo maggioritario-proporzionale. Parleremo allora di tipi puri se i sistemi misti appartengono in via esclusiva ad una delle tre categorie individuate, e di tipi ibridi se essi presentano invece caratteristiche che li associano a più di una tra quelle stesse categorie.

La separazione/fusione meccanica e la separazione/fusione territoriale sono senz'altro le due variabili fondamentali che consentono di distinguere tra loro i diversi tipi di sistemi elettorali misti. Senza rinunciare all'indispensabile parsimonia concettuale è tuttavia necessario andare più in là di quanto abbiano fatto Blais e Massicotte. Fermo restando che i due autori hanno individuato correttamente quelli che possono essere definiti i tipi principali di sistemi misti, appare utile procedere ad un'ulteriore differenziazione tra $\mathrm{i}$ tipi individuati, così da mettere in luce altri importanti aspetti della connessione maggioritarioproporzionale che altrimenti rimarrebbero in ombra ${ }^{8}$. In sostanza, si aggiungono altri due criteri discriminanti oltre a quelli già menzionati, sebbene subordinati ad essi secondo una logica di tipo tassonomico'. Così facendo, si hanno le seguenti quattro

8 Se così non fosse, si correrebbe il rischio di generare una sorta di can-gatto classificatorio, specialmente in riferimento - come si vedrà più avanti - alla categoria dei sistemi misti a correzione.

9 L'applicazione della logica tassonomica ai fini della classificazione dei sistemi elettorali in generale, e di quelli misti in particolare, è suggerita anche da Monroe (1994). 
variabili dicotomiche che consentono di selezionare tipi e sottotipi di sistemi elettorali misti ${ }^{10}:$ 1) separazione meccanica, 2) separazione territoriale, 3) dipendenza gerarchica e 4) separazione di voto.

Delle prime due si è già detto. Quanto alla dipendenza gerarchica, essa serve a far luce su una significativa differenza interna ai sistemi misti a correzione. La correzione, infatti, implica l'interdipendenza meccanica ma non ci dice se i due livelli di ripartizione dei seggi si collochino o meno in una posizione gerarchica, ovvero se uno - quello proporzionale - costituisca il livello decisivo per l'assegnazione complessiva dei seggi. Se esiste una dipendenza gerarchica si hanno i sistemi misti a correzione completa, mentre se non esiste si hanno i sistemi a semicorrezione. $\mathrm{Da}$ un altro punto di vista, diremo che il metodo della semi-correzione presenta le stesse caratteristiche del metodo della correzione, ma si riferisce più specificamente ai casi nei quali la correzione, ossia i meccanismi di compensazione proporzionalistica rispetto alla distorsione realizzatasi in sede uninominale, avviene mediante scorporo dei voti (e non dei seggi, come invece nel caso di correzione completa ${ }^{11}$. Questa ulteriore distinzione sarà chiara più avanti quando confronteremo concretamente le differenze presenti tra le due categorie.

La separazione di voto, infine, pone l'accento su un altro e non secondario aspetto della connessione tra $i$ livelli maggioritario e proporzionale di ripartizione dei seggi. Che l'elettore abbia la possibilità di esprimere un unico voto per entrambe le arene (voto fuso) $)^{12}$ o un voto per ciascuna di quelle (voto disgiunto) fa infatti una notevole differenza sia rispetto alle sue stesse scelte che rispetto alle strategie di competizione dei partiti, e, di conseguenza, può incidere anche sui risultati elettorali. Si consideri, ad esempio, il caso del voto fuso: in quanto impone una scelta univoca, esso tende a creare un dilemma per l'elettore allorché il candidato uninominale preferito non sia associato al partito preferito (o viceversa) o, anche nel caso in cui

10 Anche qui, come in precedenza, si fa riferimento ai soli tipi puri.

$11 \mathrm{Si}$ potrebbe anche introdurre una distinzione tra lo scorporo parziale e quello totale dei voti ma, nel rispetto del principio della parsimonia nel procedimento di classificazione, faremo cenno ad essa solo quando tratteremo più in dettaglio dei sistemi elettorali di Italia e Ungheria.

12 La denominazione di voto fuso è mutuata da Cox (1997), anche se quest'ultimo la utilizza non in riferimento ai sistemi elettorali misti bensì nei casi di una scelta di voto unica che lega tra loro elezioni di tipo diverso (es. presidenziali e parlamentari). 
lo fosse, non abbia chance di vittoria (la defezione da quel candidato in favore di uno più competitivo significherebbe, infatti, anche la defezione dal partito preferito, che invece potrebbe avere la possibilità di conquistare seggi). Tutto ciò ovviamente non si verifica se vi è la possibilità di esprimere un voto disgiunto, a meno che le due arene di voto e di ripartizione dei seggi siano interdipendenti come nei sistemi misti a correzio$\mathrm{ne}^{13}$. Per quanto riguarda i partiti, poi, l'esistenza del voto disgiunto - e quindi di due distinte arene di competizione - significa la possibilità di diversificare le proprie strategie, ad esempio apparentandosi ad altri nella parte maggioritaria e correndo da soli nella parte proporzionale - il che è evidentemente impedito nel caso di voto fuso. Insomma, già da questi brevi cenni si può comprendere l'importanza che la variabile separazione di voto assume nei sistemi elettorali misti e, quindi, la necessità che essa venga utilizzata ai fini della classificazione degli stessi sistemi misti.

In figura 1 sono riportati schematicamente tutto il procedimento classificatorio sin qui descritto nonché i tipi e sotto-tipi di sistemi misti individuati ${ }^{14}$.

A questo punto è possibile passare all'analisi empirica dei sistemi elettorali misti. Prima di vedere più in dettaglio come siano in concreto congegnati i sistemi a combinazione, a correzione e a coesistenza, è però necessario specificare che l'universo empirico di riferimento qui considerato è costituito dai sistemi elettorali misti utilizzati per l'elezione della Camera bassa (o, nel caso, dell'unica Camera esistente) in regimi democratici di antico consolidamento o ancora in transizione (ma che superano $i$ requisiti minimi per definirsi tali) ${ }^{15}$ - escludendo quelle elezioni che siano state caratterizzate, secondo gli osservatori internazionali, da irregolarità diffuse. Come in Lijphart (1994), ven-

$13 \mathrm{Nel}$ qual caso alcune considerazioni fatte per il voto fuso potrebbero essere di nuovo valide. Si tenga conto, comunque, che le strategie di voto (per coloro che lo vogliono utilizzare al meglio) variano in conseguenza di tali e tante condizioni che è qui difficile riassumerle in poche parole. Per quanto ipotizzato è implicita dunque la clausola ceteris paribus.

14 I tipi e sotto-tipi individuati sono in ogni caso solo quelli che hanno un referente empirico.

15 Tra i regimi non democratici che impiegano sistemi elettorali misti troviamo Armenia, Azerbajan, Senegal e Tunisia. Anche la Jugoslavia nel 1992, in piena guerra, utilizzò un sistema misto (Kasapovic 1995) e così lo Zimbabwe nel 1980, con la parte maggioritaria (1/5 dei seggi) riservata alla minoranza bianca e la parte proporzionale $(4 / 5)$ alla maggioranza nera (Lijphart 1986). 


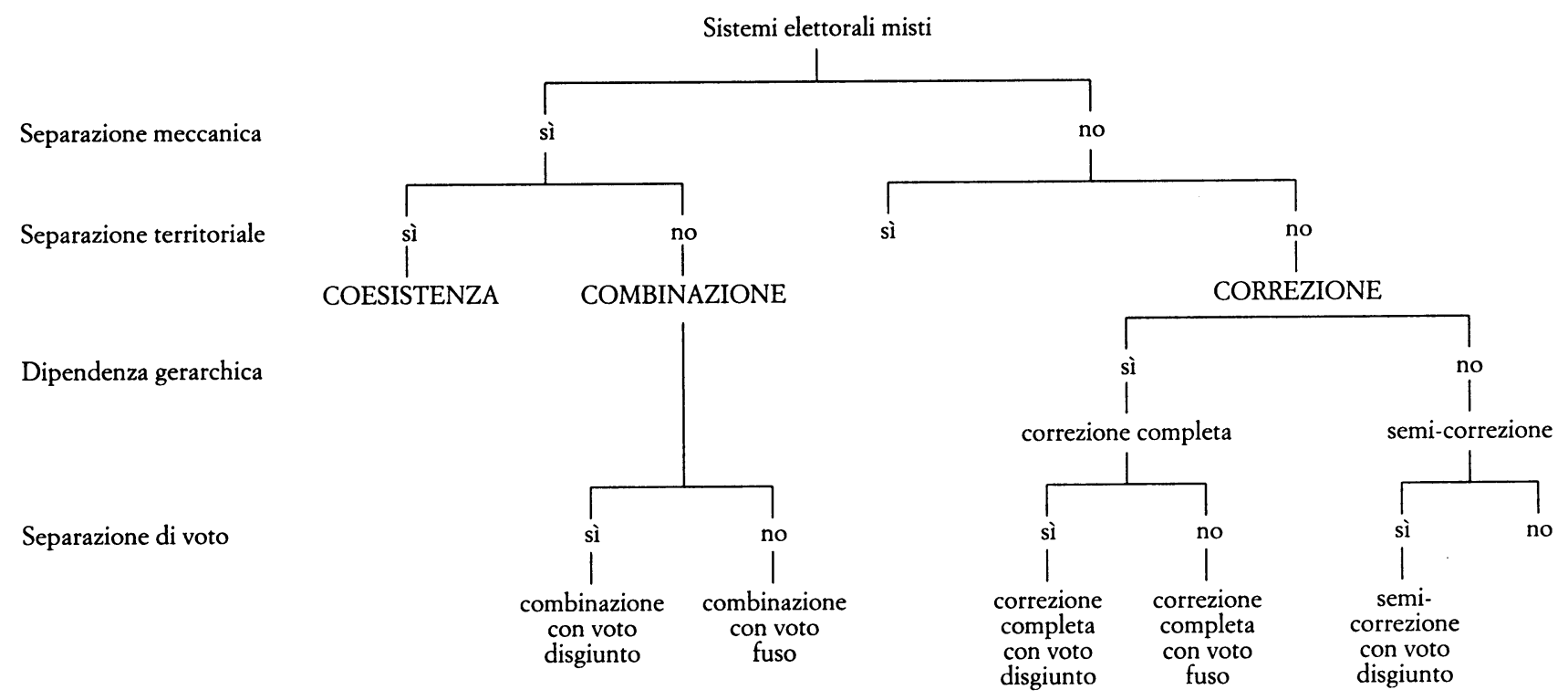

Legenda: in maiuscolo sono indicati i tipi puri principali.

FIG. 1. Classificazione dei sistemi elettorali misti. 
TAB. 1. I sistemi elettorali misti nei regimi democratici

\begin{tabular}{|c|c|c|c|}
\hline $\begin{array}{l}\text { Sistema } \\
\text { elettorale }\end{array}$ & $\begin{array}{l}\text { Periodo } \\
\text { applicazione }\end{array}$ & $\begin{array}{c}\mathrm{N} \mathrm{di} \\
\text { elezioni }\end{array}$ & Tipo $^{1}$ \\
\hline Albania I & 1992 & 1 & CORREZIONE completa con voto fuso \\
\hline Albania II & 1996- & 1 & COMBINAZIONE con voto fuso \\
\hline Bulgaria & 1990 & 1 & COMBINAZIONE con voto separato \\
\hline Corea (Sud) & 1996- & 1 & COMBINAZIONE con voto fuso \\
\hline Croazia $^{2}$ & 1995. & 1 & COMBINAZIONE con voto separato \\
\hline Danimarca & 1918 & 1 & Coesistenza + Correzione \\
\hline Francia & 1951.56 & 2 & COESISTENZA \\
\hline Georgia I & 1992 & 1 & COMBINAZIONE con voto separato \\
\hline Georgia II & 1995- & 1 & COMBINAZIONE con voto separato \\
\hline Germania I & 1949 & 1 & CORREZIONE completa con voto fuso \\
\hline Germania II & 1953 & 1 & $\begin{array}{l}\text { CORREZIONE completa con voto se- } \\
\text { parato }\end{array}$ \\
\hline Germania III & $1957-83$ & 8 & $\begin{array}{l}\text { CORREZIONE completa con voto se- } \\
\text { parato }\end{array}$ \\
\hline Germania IV & 1987 & 1 & $\begin{array}{l}\text { CORREZIONE completa con voto se- } \\
\text { parato }\end{array}$ \\
\hline Germania V & $1990-$ & 2 & $\begin{array}{l}\text { CORREZIONE completa con voto se- } \\
\text { parato }\end{array}$ \\
\hline Giappone & 1996- & 1 & COMBINAZIONE con voto separato \\
\hline Italia & $1994-$ & 2 & Semi-CORREZIONE con voto separato \\
\hline Lituania I & 1992 & 1 & COMBINAZIONE con voto separato \\
\hline Lituania II & 1996- & 1 & COMBINAZIONE con voto separato \\
\hline Messico $^{3}$ & 1994- & 1 & COMBINAZIONE con voto separato \\
\hline Nuova Zelanda & 1996- & 1 & $\begin{array}{l}\text { CORREZIONE completa con voto se- } \\
\text { parato }\end{array}$ \\
\hline Russia & 1993- & 2 & COMBINAZIONE con voto separato \\
\hline Taiwan & 1996- & 1 & COMBINAZIONE con voto fuso \\
\hline Ungheria I & 1990 & 1 & $\begin{array}{l}\text { Combinazione }+ \text { Semi-Correzione con } \\
\text { voto separato }\end{array}$ \\
\hline Ungheria II & 1994- & 1 & $\begin{array}{l}\text { Combinazione }+ \text { Semi-Correzione con } \\
\text { voto separato }\end{array}$ \\
\hline Venezuela & 1993- & 1 & $\begin{array}{l}\text { CORREZIONE completa con voto se- } \\
\text { parato }\end{array}$ \\
\hline
\end{tabular}

Note:

1 In maiuscoletto sono indicati i tipi puri principali, in corsivo i tipi ibridi.

2 Sebbene tenutesi con un sistema elettorale simile a quello utilizzato nel 1995, le elezioni croate del 1992 non sono qui considerate poiché concomitanti con la guerra allora in corso.

3 Il Messico ha adottato vari sistemi elettorali misti per l'elezione della Camera bassa sin dal 1963. Tuttavia, è solo con quello vigente a partire dal 1994 che la competizione si è effettivamente aperta alle forze di opposizione.

Fonti: vedi tabb. 2, 3 e 4. 
gono inoltre distinti tra loro quei sistemi elettorali di uno stesso paese nei quali siano intervenuti cambiamenti relativi alla formula o - se maggiori del $20 \%$ - ad altre tre variabili quali la dimensione media dei collegi, l'ampiezza parlamentare e la soglia legale di accesso alla rappresentanza.

Nel rispetto dei limiti appena menzionati, i sistemi elettorali misti rispondenti alla definizione riportata nella sezione precedente risultano essere ben 25 - adottati in 17 paesi diversi - di cui 14 erano vigenti alla fine del 1996. La tabella 1 dà conto di quali siano i 25 sistemi elettorali misti considerati in questa analisi e di ognuno di essi rileva il periodo temporale e il numero di elezioni in cui è stato applicato, nonché il tipo di raccordo proporzionale-maggioritario che adotta.

Nelle tre sezioni che seguono procederemo ad una descrizione più puntuale dei nostri 25 casi, a cominciare dai sistemi a combinazione, per passare poi a quelli a correzione, e concludere con i sistemi misti a coesistenza e i tipi ibridi. Nelle tabelle 2 4 sintetizzeremo le caratteristiche principali di ciascun sistema elettorale misto sulla base delle seguenti variabili: 1) il numero di voti a disposizione dell'elettore $\left.{ }^{16} ; 2\right)$ l'ampiezza dell'assemblea parlamentare; e poi, in riferimento a ciascuno dei due livelli di assegnazione dei seggi: 3) la formula elettorale; 4) il numero medio di seggi; 5) la percentuale relativa del numero medio di seggi; 6) il numero medio di collegi/circoscrizioni; 7) la dimensione media di collegi/circoscrizioni ${ }^{17}$; 8) l'entità di soglie legali per l'accesso alla rappresentanza, se presenti.

\section{I sistemi misti a combinazione}

La categoria più densa di casi empirici è quella dei sistemi misti a combinazione (tab. 2). Come detto in precedenza, questi

16 Si intendono solo i voti il conteggio dei quali è rilevante ai fini dell'assegnazione dei seggi tra i partiti (seat-relevant votes). Altre espressioni di scelta, ad esempio il voto di preferenza, sono escluse.

17 I collegi (uninominali) e le circoscrizioni (plurinominali) di cui si parla sono solo quelli in cui ha luogo l'effettiva distribuzione dei seggi. Si assiste infatti anche a casi di ritagli territoriali «fittizi»: ne è un esempio il sistema elettorale della Slovenia, che prevede l'espressione del voto a un candidato presente nel proprio collegio (falsamente uninominale), ma la ripartizione (proporzionale) dei seggi sulla base della somma dei voti raccolti dai candidati dello stesso partito nell'ambito di una circoscrizione che comprende più di uno di quei collegi. 
TAB. 2. Caratteristiche principali di dodici sistemi elettorali misti a combinazione

\begin{tabular}{|c|c|c|c|c|c|c|c|c|c|}
\hline $\begin{array}{l}\text { Sistema } \\
\text { Elettorale }\end{array}$ & $\begin{array}{c}\text { Numero } \\
\text { di voti }\end{array}$ & $\begin{array}{l}\text { Ampiezza } \\
\text { assemblea } \\
\text { parlament. }\end{array}$ & $\begin{array}{l}\text { Livelli di } \\
\text { ripartizione } \\
\text { dei seggi }\end{array}$ & $\begin{array}{l}\text { Formula } \\
\text { elettorale }\end{array}$ & $\begin{array}{c}\text { Numero } \\
\text { medio } \\
\text { di seggi }\end{array}$ & $\begin{array}{l}\% \text { di seggi } \\
\text { per ciascun } \\
\text { livello }\end{array}$ & $\begin{array}{c}\text { Numero } \\
\text { medio } \\
\text { collegi }\end{array}$ & $\begin{array}{c}\text { Dimensione } \\
\text { media } \\
\text { dei collegi }\end{array}$ & $\begin{array}{l}\text { Soglie } \\
\text { legali }\end{array}$ \\
\hline \multicolumn{10}{|l|}{ Con voto fuso: } \\
\hline \multirow[t]{2}{*}{ Albania II } & 1 & 140 & MG & majority-runoff & 115 & 82,1 & 115 & 1 & \\
\hline & & & PR-naz. & 1 & 25 & 17,9 & 1 & 25 & $4 \%$ naz. \\
\hline \multirow[t]{2}{*}{ Corea (Sud) } & 1 & 299 & MG & plurality & 253 & 84,6 & 253 & 1 & \\
\hline & & & PR-naz. & LR-Hare & 46 & 15,4 & 1 & 46 & $5 \%$ naz. \\
\hline \multirow[t]{2}{*}{ Taiwan } & 1 & 334 & MG & Sntv & 234 & 70,1 & 58 & 4,0 & \\
\hline & & & PR-naz. & LR-Hare & $100^{2}$ & 29,9 & 1 & 100 & $5 \%$ naz. \\
\hline \multicolumn{10}{|l|}{ Con voto disgiunto: } \\
\hline \multirow[t]{2}{*}{ Bulgaria } & 2 & 400 & MG & majority-runoff & 200 & 50,0 & 200 & 1 & \\
\hline & & & PR-reg. & d'Hondt & 200 & 50,0 & 28 & 7,1 & $4 \%$ naz. \\
\hline \multirow[t]{3}{*}{ Croazia } & 2 & 127 & MG & plurality & 353 & 27,6 & 33 & 1,0 & \\
\hline & & & PR-naz. & d'Hondt & 80 & 63,0 & 1 & 80 & $5 \%-8 \%-11 \%$ naz. 5 \\
\hline & & & PR-est. & d'Hondt & 124 & 9,4 & 1 & 12 & $5 \%-8 \%-11 \%^{5}$ \\
\hline \multirow[t]{3}{*}{ Georgia I } & 2 & 235 & MG & majority-runoff & 856 & 36,2 & 85 & 1 & \\
\hline & & & PR-reg. & Hare & 70 & 29,8 & 10 & 10 & - \\
\hline & & & PR-naz. & d'Hondt & 80 & 34,0 & 1 & 80 & - \\
\hline \multirow[t]{2}{*}{ Georgia II } & 2 & 235 & MG & majority-runoff & 85 & 36,2 & 85 & 1 & \\
\hline & & & PR-reg. & d'Hondt & 150 & 63,8 & 10 & 15 & $5 \%$ naz. \\
\hline \multirow[t]{2}{*}{ Giappone } & 2 & 500 & MG & plurality & 300 & 60,0 & 300 & 1 & \\
\hline & & & PR-reg. & d'Hondt & 200 & 40,0 & 11 & 18,1 & - \\
\hline
\end{tabular}




\begin{tabular}{|c|c|c|c|c|c|c|c|c|c|}
\hline Lituania I & 2 & 141 & $\begin{array}{l}\text { MG } \\
\text { PR-naz. }\end{array}$ & $\begin{array}{l}\text { majority-runoff } \\
\text { LR-Droop }\end{array}$ & $\begin{array}{l}71 \\
70\end{array}$ & $\begin{array}{l}50,4 \\
49,6\end{array}$ & $\begin{array}{r}71 \\
1\end{array}$ & $\begin{array}{r}1 \\
70\end{array}$ & $4 \%$ naz. \\
\hline & & & PR-naz. & LR-Droop & 70 & 49,6 & 1 & 70 & $5 \%-7 \%$ naz. ${ }^{7}$ \\
\hline Messico 8 & 2 & 500 & MG & plurality & 300 & 60,0 & 300 & 1 & \\
\hline Russia & 2 & 450 & $\begin{array}{l}\text { MG } \\
\text { PR-naz. }\end{array}$ & $\begin{array}{l}\text { plurality } \\
\text { LR-Hare }\end{array}$ & $\begin{array}{l}225 \\
225\end{array}$ & $\begin{array}{l}50,0 \\
50,0\end{array}$ & $\begin{array}{r}225 \\
1\end{array}$ & $\begin{array}{r}1 \\
225\end{array}$ & $5 \%$ naz. \\
\hline
\end{tabular}

Note:

1 «I 25 seggi sono ripartiti sulla base della percentuale di voti conseguita dai singoli partiti sul territorio nazionale: un partito con il $4 \%$ ottiene un seggio, un partito con il $20 \%$ ne ottiene cinque. I seggi non ripartiti attraverso i quozienti interi sono assegnati al partito che ha ottenuto il maggior numero di seggi nei collegi uninominali» (The Norvegian Helsinki Committee 1996a).

$2 \mathrm{Di}$ cui venti rappresentanti della comunità cinese d'oltremare.

3 Di cui sette rappresentanti delle minoranze nazionali: quattro vengono eletti in appositi collegi uninominali tra gli esponenti delle comunità 1) italiana, 2) ungherese, 3) ceca e slovacca, 4) rutena, ucraina, tedesca e austriaca; tre vengono eletti in un collegio plurinominale, ma sempre con la regola plurality, tra gli esponenti della comunità serba.

4 Si tratta dei rappresentanti che vengono eletti, sulla base di liste speciali, dai croati residenti all'estero.

5 Le tre soglie si riferiscono, rispettivamente, a singoli partiti $(5 \%)$, a liste di due partiti apparentati $(8 \%)$, a liste di tre o più partiti apparentati $(11 \%)$.

6 Di cui un seggio è riservato all'elezione - in un collegio unico coincidente con l'intero territorio nazionale - del presidente dell'assemblea parlamentare.

7 Le due soglie si riferiscono, rispettivamente, a singoli partiti (5\%) e a liste di due o più partiti apparentati $(7 \%)$.

8 Nessun partito può ricevere in tutto più di 300 seggi (o 315 se ha più del $60 \%$ dei voti).

Fonti: Albania II: Inter-Parliamentary Union (Ipu), The Norwegian Helsinki Committee (1996a); Corea (Sud): Ipu, Morriss (1996); Taiwan: Hsieh e Niou (1996). Bulgaria: Koulov (1995), Crampton (1991); Croazia: The Law on Elections of Representatives to the Parliament of the Republic of Croatia (Amended Text) (1995), The Law on Voting Units for the House of Representatives of the Parliament of the Republic of Croatia (1995); Georgia I e II: Ipu, The Norwegian Helsinki Committee (1995), Allison, Kukhianidze e Matsaberidze (1993), Allison (1996); Giappone: Ipu, Shiratori (1995); Lituania I e II: Ipu; Messico: Ley Electoral Mexicana (reformada, 1990-1994), Craig e Cornelius (1995), Balinski e Ramirez Gonzalez (1996); Russia: Ipu, The Norwegian Helsinki Committee (1996b), International Foundation for. Electoral Systems (1995), Moser (1995). 
sistemi prevedono la separazione meccanica ma non territoriale dei livelli maggioritario e proporzionale di ripartizione dei seggi.

Il sistema elettorale utilizzato in Russia a partire dalle elezioni del 1993 è esemplificativo del funzionamento di un sistema misto a combinazione.

Il territorio nazionale russo è diviso in 225 collegi uninominali in ciascuno dei quali viene eletto il candidato che ottiene il maggior numero di voti (plurality). Altri 225 seggi vengono poi attribuiti - in modo del tutto separato - tra liste concorrenti in una circoscrizione coincidente con l'intero territorio nazionale mediante il metodo del quoziente naturale e dei più alti resti (LR-Hare), con la clausola che le stesse liste abbiano ottenuto almeno il 5\% di voti validi. In sostanza, il 50\% dei seggi complessivi viene assegnato sulla base del sistema maggioritario-uninominale (a turno unico) e l'altro $50 \%$ su base proporzionale in modo indipendente dal punto di vista meccanico.

Il sistema elettorale russo prevede inoltre che l'elettore possa esprimere la propria preferenza sia per un candidato di collegio che per una lista proporzionale - è dunque un sistema misto a combinazione con voto disgiunto. Sotto questo profilo, la maggioranza degli altri sistemi a combinazione è conforme ad esso, ma non così i casi Albania II, Corea del Sud e Taiwan nei quali il voto è fuso. Quest'ultimi permettono all'elettore di votare soltanto per un candidato di collegio, e così facendo la preferenza espressa vale automaticamente anche per la lista alla quale quel candidato appartiene e che concorre per l'assegnazione dei seggi proporzionali. La «fusione» del voto, è bene ribadirlo, rappresenta una contaminazione tra i due livelli quanto alle logiche di scelta degli elettori e alle strategie di competizione dei partiti che in presenza del voto disgiunto - sempre in sistemi a combinazione - invece non si verifica.

A prescindere dalla separazione/fusione del voto, gli altri sistemi misti a combinazione variano rispetto a quello russo per le formule elettorali impiegate, o per l'entità della soglia di sbarramento, o, ancora, per il numero e la dimensione delle circoscrizioni proporzionali - caratteristiche che non hanno direttamente a che fare con la natura mista di questi sistemi elettorali, ma che ne influenzano senza dubbio gli effetti complessivi.

Per ciò che concerne la formula elettorale maggioritaria, in sei casi (Albania II, Bulgaria, Georgia I e II, Lituania I e II) viene utilizzato il sistema maggioritario a doppio turno - con la specificazione che al secondo turno accedono solo i due candi- 
dati con più voti (majority-runoff) -, mentre in cinque casi (Corea, Croazia, Giappone, Messico e appunto Russia) vige il metodo plurality. Taiwan adotta invece il sistema del singolo voto non trasferibile (Sntv) in collegi plurinominali (con una dimensione media pari a 4). Il Sntv dispone che in ciascun collegio l'elettore voti per un unico candidato, ma che vengano eletti tanti candidati (ovviamente quelli con il più alto numero di voti) quanti sono i seggi da assegnare in quel collegio ${ }^{18}$. A livello proporzionale la variabilità della formula tra i nostri sistemi a combinazione è anche maggiore. Il metodo d'Hondt è applicato in Bulgaria, Croazia (sia per le liste nazionali che per le liste speciali oggetto del voto dei residenti esteri), Georgia II e Giappone; il LR-Hare in Corea del Sud, Taiwan, Messico e Russia; il quoziente con correttore +1 e più alti resti (LR-Dro$\mathrm{op}^{19}$ ) in Lituania I e II. Il sistema elettorale Georgia I, sempre riguardo alla ripartizione proporzionale dei seggi, è a due livelli (two-tiers): a livello regionale viene assegnato un numero di seggi pari ai quozienti naturali (Hare) interi dei singoli partiti concorrenti ${ }^{20}$; i voti e i seggi residuali confluiscono poi in una circoscrizione unica nazionale dove si applica il metodo d'Hondt. Per l'Albania II vige invece un sistema particolare, basato su un quoziente predeterminato - il $4 \%$ dei voti validi, pari all'entità della soglia di sbarramento - e sull'assegnazione dei seggi-resto

18 In letteratura non c'è accordo circa l'appartenenza del Sntv alla categoria delle formule maggioritarie. Lijphart $(1994,18)$ sostiene che le formule maggioritarie sono quelle caratterizzate dalla corrispondenza tra il numero degli eletti in un collegio e il numero dei voti a disposizione degli elettori; e che, pertanto, il Sntv non sia tra quelle prevede infatti un solo voto ma sempre più di un eletto nel collegio. Al Sntv Lijphart preferisce riservare la denominazione di formula semi-proporzionale (come già Lakeman 1970), anche in considerazione del fatto che tale sistema consente, nei singoli collegi plurinominali, una qualche forma di rappresentanza delle minoranze. Cox (1997, 58-59 e 100-101) ritiene invece ambigua proprio l'etichetta semi-proporzionale in riferimento al Sntv. Secondo Cox, infatti, la meccanica del Sntv è identica a quella del plurality: vince chi ottiene più voti. La sola differenza tra i due sistemi risiede nella dimensione dei collegi, che con il plurality è pari a uno, mentre con il Sntv è variabile e sempre superiore ad uno. Per Cox, in sostanza, il Sntv è una formula maggioritaria ed è così che qui lo consideriamo.

19 Come in Lijphart (1994), la dizione Droop è qui sinonimo di quella Hagenbach-Bishoff.

20 Il caso Georgia I è in verità più complicato, configurandosi come sistema a punti (point-system). Gli elettori dispongono, per la parte proporzionale, di un voto di tipo ordinale, attribuiscono cioè dei «punti» alle liste presenti a seconda del loro ordine di preferenza. L'assegnazione dei seggi, fermo restando l'impiego delle formule come si è detto, procede quindi sulla base dei punti di ciascun partito. Per maggiori dettagli si rinvia a Allison, Kukhianidze e Matsaberidze (1993). 
al partito che ha visto eletto il più alto numero di candidati in sede maggioritaria.

Tutti i sistemi a combinazione qui considerati, con l'eccezione di Georgia I e Giappone, prevedono poi una soglia legale di sbarramento per l'accesso alla distribuzione proporzionale dei seggi. Essa è fissata al 4 o $5 \%$, scendendo all'1,5 (ma dei votanti, il che significa che è certamente superiore se calcolata sui voti validi) solo per il Messico. Il Giappone non ha, come detto, una soglia legale, ma in realtà ne ha una di fatto che si attesta mediamente proprio intorno al $5 \%$ dei voti ${ }^{21}$ - in conseguenza del fatto che la ripartizione dei seggi ha luogo in circoscrizioni regionali con un'ampiezza media pari a $18,1^{22}$. La soglia effettiva in Georgia I è invece molto bassa nonostante le circoscrizioni regionali siano persino più piccole di quelle del Giappone: la presenza di un livello nazionale dove vengono recuperati i voti non utilizzati in sede regionale garantisce, infatti, anche ai partiti con modeste percentuali di voto di essere rappresentati. A proposito della dimensione dei collegi, è da notare inoltre una forte corrispondenza tra l'adozione di soglie legali di sbarramento e la ripartizione dei seggi proporzionali su base nazionale. Gli unici casi devianti sono Bulgaria e Georgia II, che prevedono una soglia legale nazionale (rispettivamente $4 \mathrm{e}$ $5 \%$ ) e l'assegnazione dei seggi su base regionale. Ne deriva che in questi ultimi sistemi le circoscrizioni più piccole impongano in realtà una soglia di fatto ben più alta di quella prevista a livello nazionale.

Prima di concludere con i sistemi a combinazione, è opportuno sottolineare altri due elementi di differenziazione che hanno ora a che fare in modo diretto con la natura mista di questi sistemi elettorali. Essi fanno riferimento l'uno, ancora una volta, alla struttura del voto rispetto ai due livelli di ripartizione dei seggi e l'altro al «dosaggio» tra seggi maggioritari e seggi proporzionali.

Ritorniamo, in primo luogo, alla questione del voto fuso e del voto disgiunto che si lega significativamente con le soglie di sbarramento adottate in sede proporzionale di cui si è appena

21 Si tratta della cosiddetta soglia di esclusione, ossia della massima percentuale di voti che un partito può ottenere senza ricevere seggi. Ciò significa che un partito con una percentuale di voti inferiore a quella potrebbe comunque conquistare seggi.

22 È noto che l'ampiezza della circoscrizione è correlata negativamente all'entità della soglia effettiva di accesso alla rappresentanza. 
detto. Si considerino i sistemi a combinazione con voto fuso: appare evidente che in questi casi la soglia nazionale del $4 \%$ o $5 \%$ ha un'influenza maggiore in confronto ai casi di combinazione con voto disgiunto, anche se la soglia è di pari entità. Immaginiamo, infatti, cosa può succedere ad una formazione che abbia un consenso potenziale (cioè di voto sincero) intorno al 6-7\% e che intenda competere da sola. In presenza del voto disgiunto otterrebbe seggi grazie alla parte proporzionale perché: 1) gli elettori non hanno timore di «sprecare» il loro voto (proporzionale) e, quindi, 2) votano sinceramente la lista che così supera la soglia. Con il voto fuso, però, quella stessa formazione rischierebbe forti defezioni strategiche e potrebbe rimanere priva di rappresentanza. A causa della commistione delle arene competitive, infatti, i suoi elettori, che hanno un'unica scelta, o 1) rinunciano a far contare (e quindi sprecano) il loro voto ai fini dell'elezione del candidato di collegio (posto che quello della lista preferita non abbia possibilità di vincere) così da avvantaggiare il partito in sede proporzionale, o 2) votano strategicamente per un candidato di collegio competitivo defezionando però dal partito, che vede in tal modo diminuire le possibilità di superare la soglia proporzionale. Insomma, è probabile che la nostra formazione politica, pur mantenendo una quota di elettori fedeli, perda una quota di elettori strategici decisiva per il conseguimento della rappresentanza parlamentare $e^{23}$. E questo sarebbe un effetto da imputare al voto fuso.

Il secondo aspetto di rilievo che preme qui segnalare quale elemento di diversificazione nell'ambito dei sistemi misti a combinazione è la proporzione relativa di seggi maggioritari e seggi uninominali. In Russia, come abbiamo visto, e in Bulgaria è identica. Anche nei due sistemi della Lituania è pressoché tale (solo un seggio in più viene assegnato in sede maggioritaria). Tra quelli con una prevalenza della quota proporzionale troviamo, in ordine crescente, Georgia I e II $(63,8 \%)$ e Croazia $(72,4 \%)$. All'opposto, quelli con una prevalenza della quota maggioritaria sono Giappone e Messico (entrambi 60\%), Taiwan (70,1\%), Albania II $(82,1 \%)$ e Corea del Sud $(84,6 \%)$. Va da sé che, ceteris paribus, quanto più alto è il numero dei seggi assegnati mediante

23 È dunque presente un forte incentivo all'aggregazione con altre forze. Si badi però che l'aggregazione verrebbe pagata al prezzo della rinuncia alla propria visibilità, cosa che non accadrebbe nel caso di voto disgiunto - essendo l'arena di competizione proporzionale separata da quella maggioritaria. 
formula maggioritaria, tanto più il sistema elettorale misto si presenta sulla carta distorsivo e selettivo.

\section{I sistemi misti a correzione}

I sistemi misti a correzione sono quelli che non prevedono né la separazione meccanica né la separazione territoriale tra $\mathrm{i}$ livelli maggioritario e proporzionale di ripartizione dei seggi. La tabella 3 illustra le principali caratteristiche di nove sistemi elettorali che appartengono a questo tipo, distinguendoli preliminarmente tra sistemi a correzione completa - con voto fuso o disgiunto - e sistemi a semi-correzione.

Il caso paradigmatico dei sistemi a correzione completa è quello tedesco, se non altro perché tra tutti quelli considerati ha la maggiore durata di applicazione. In realtà, dal 1949 ad oggi esso ha subito importanti modifiche - tanto che, secondo i criteri mutuati da Lijphart, è necessario parlare di 5 sistemi elettorali diversi. Nel 1953, rispetto al 1949, si passa dalla possibilità di esprimere un solo voto al voto disgiunto ${ }^{24}$; inoltre, la soglia di sbarramento del $5 \%$ non si applica più a livello di circoscrizioni regionali bensì in sede nazionale. Nel 1957 anche l'effettiva distribuzione dei seggi tra le liste passa dal livello regionale al livello nazionale; è altresì modificata la clausola per l'accesso alla ripartizione proporzionale dei seggi: non più il $5 \%$ o 1 seggio uninominale, bensì il $5 \%$ o 3 seggi uninominali. Nel 1987 viene riformata la formula proporzionale: il metodo d'Hondt viene sostituito dal metodo del quoziente naturale e dei più alti resti. Nel 1990, infine, l'unificazione tra Germania occidentale e Germania orientale comporta un significativo aumento del numero dei seggi complessivi da attribuire.

Ciò che invece è rimasto immutato del sistema tedesco durante tutto lo stesso periodo è quella che può essere definita la sua caratteristica principale: la decisività del livello proporzionale per stabilire $\mathrm{i}$ rapporti di forza parlamentare dei partiti. Una caratteristica che è propria di tutti i sistemi misti a correzione completa.

24 Il sistema tedesco del 1949 costituisce la traduzione concreta di quello che è noto come metodo Geyerhahn, risalente all'inizio del secolo. Già l'introduzione del voto disgiunto nel 1953 rappresentava però uno scostamento dal modello originario. Su questo argomento si veda Carducci (1994). 
In Germania, come già in Russia, il territorio nazionale è suddiviso in un numero di collegi uninominali pari alla metà della dimensione dell'assemblea parlamentare. In ciascuno di essi vince il candidato che ottiene più voti. Tuttavia, a differenza che in Russia, la ripartizione proporzionale dei seggi risulta determinante per le formazioni politiche in competizione: essa, infatti, viene effettuata con riferimento al totale dei seggi e non alla metà di essi ${ }^{25}$. Dalla quota di seggi spettante a ciascun partito sulla base della distribuzione proporzionale viene poi detratto (scorporato) il numero di seggi che quello stesso partito ha già conseguito in sede di collegi uninominali - fatta salva la circostanza che quest'ultima cifra sia superiore, come talvolta accaduto, alla precedente, nel qual caso il partito conserva il surplus di seggi, i cosiddetti Überbangmandaten ${ }^{26}$.

I sistemi elettorali attualmente vigenti in Nuova Zelanda e in Venezuela sono molto simili a quello tedesco. La Nuova Zelanda si differenzia principalmente per l'adozione della formula Saint-Laguë e per la presenza di un maggior numero di seggi maggioritari - conseguente alla riserva di 5 collegi uninominali per la rappresentanza della minoranza Maori. In Venezuela la ripartizione proporzionale dei seggi avviene, come nei sistemi Germania I e II, sulla base del metodo d'Hondt in circoscrizioni regionali e non vi è una soglia legale di sbarramento. Ė previsto inoltre un ulteriore livello compensativo di attribuzione dei seggi, per quanto limitato in termini numerici.

Tra i sistemi a correzione completa con voto fuso, oltre al già citato sistema tedesco del 1949 troviamo quello albanese adottato nelle elezioni del 1992. In questo caso i seggi uninominali sono attribuiti con il sistema del doppio turno chiuso. $\grave{E}$ poi da notare una certa prevalenza della parte maggioritaria ( $71,4 \%$ dei seggi complessivi) che può in teoria favorire il fenomeno del surplus di seggi - specie se un partito è in grado di espugnare un ampio numero di collegi uninominali - e quindi limitare le capacità compensative intrinseche all'impianto a correzione di questo tipo di sistema elettorale ${ }^{27}$.

25 In tabella 3 si può notare, infatti, che la dimensione media dei collegi della parte proporzionale (quando la ripartizione dei seggi è effettuata a livello nazionale) è coincidente con l'ampiezza dell'assemblea parlamentare.

26 L'assegnazione di seggi in sovrannumero si è verificata nelle seguenti elezioni (tra parentesi il numero di tali seggi): 1949 (2), 1953 (3), 1957 (3), 1961 (5), 1980 (1), 1983 (2), 1987 (1), 1990 (6), 1994 (16) (Cole 1995).

27 Nel caso specifico delle elezioni albanesi del 1992 tale fenomeno non si è però verificato, perché nessun partito ha dominato la competizione maggioritaria. 
TAB. 3. Caratteristiche principali di nove sistemi elettorali misti a correzione

\begin{tabular}{|c|c|c|c|c|c|c|c|c|c|}
\hline $\begin{array}{l}\text { Sistema } \\
\text { Elettorale }\end{array}$ & $\begin{array}{l}\text { Numero } \\
\text { di voti }\end{array}$ & $\begin{array}{l}\text { Ampiezza } \\
\text { assemblea } \\
\text { parlament. }\end{array}$ & $\begin{array}{l}\text { Livelli di } \\
\text { ripartizione } \\
\text { dei seggi }\end{array}$ & $\begin{array}{l}\text { Formula } \\
\text { elettorale }\end{array}$ & $\begin{array}{c}\text { Numero } \\
\text { medio } \\
\text { di seggi }\end{array}$ & $\begin{array}{l}\% \text { di seggi } \\
\text { per ciascun } \\
\text { livello }\end{array}$ & $\begin{array}{l}\text { Numero } \\
\text { medio } \\
\text { collegi }\end{array}$ & $\begin{array}{c}\text { Dimensione } \\
\text { media } \\
\text { dei collegi }\end{array}$ & $\begin{array}{l}\text { Soglie } \\
\text { legali }\end{array}$ \\
\hline \multicolumn{10}{|c|}{$\begin{array}{l}\text { A correzione completa } \\
\text { con voto fuso: }\end{array}$} \\
\hline Albania I & 1 & 140 & $\begin{array}{l}\text { MG } \\
\text { PR-naz. }\end{array}$ & $\begin{array}{l}\text { majority-runoff } \\
\text { LR-Hare }^{1}\end{array}$ & $\begin{array}{r}100 \\
40\end{array}$ & $\begin{array}{l}71,4 \\
29,6\end{array}$ & $\begin{array}{r}100 \\
1\end{array}$ & $\begin{array}{c}1 \\
140\end{array}$ & $4 \%$ naz. \\
\hline Germania I & 1 & 402 & $\begin{array}{l}\text { MG } \\
\text { PR-reg. }\end{array}$ & $\begin{array}{l}\text { plurality } \\
\text { d'Hondt }\end{array}$ & $\begin{array}{l}240^{2} \\
162\end{array}$ & $\begin{array}{l}59,7 \\
30,3\end{array}$ & $\begin{array}{r}240 \\
11\end{array}$ & $\begin{array}{c}1 \\
36,6\end{array}$ & $\begin{array}{l}5 \% \text { reg. o } 1 \text { seggio } \\
\text { plurality }\end{array}$ \\
\hline \multicolumn{10}{|c|}{$\begin{array}{l}\text { A correzione completa } \\
\text { con voto disgiunto: }\end{array}$} \\
\hline Germania II & 2 & 487 & $\begin{array}{l}\text { MG } \\
\text { PR-reg. }\end{array}$ & $\begin{array}{l}\text { plurality } \\
\text { d'Hondt }\end{array}$ & $\begin{array}{l}242 \\
245\end{array}$ & $\begin{array}{l}49,7 \\
50,3\end{array}$ & $\begin{array}{r}242 \\
9\end{array}$ & $\begin{array}{c}1 \\
54,1\end{array}$ & $\begin{array}{c}5 \% \text { naz. o } 1 \text { seggio } \\
\text { plurality }\end{array}$ \\
\hline Germania III & 2 & 496,9 & $\begin{array}{l}\text { MG } \\
\text { PR-naz. }\end{array}$ & $\begin{array}{l}\text { plurality } \\
\text { d'Hondt }\end{array}$ & $\begin{array}{l}247,7 \\
249,2\end{array}$ & $\begin{array}{l}49,8 \\
50,2\end{array}$ & $\begin{array}{c}247,7 \\
1\end{array}$ & $\begin{array}{c}1 \\
496,9\end{array}$ & $\begin{array}{c}5 \% \text { naz. o } 3 \text { seggi } \\
\text { plurality }\end{array}$ \\
\hline Germania IV & 2 & 497 & $\begin{array}{l}\text { MG } \\
\text { PR-naz. }\end{array}$ & $\begin{array}{l}\text { plurality } \\
\text { LR-Hare }\end{array}$ & $\begin{array}{l}248 \\
249\end{array}$ & $\begin{array}{l}49,9 \\
50,1\end{array}$ & $\begin{array}{r}248 \\
1\end{array}$ & $\begin{array}{c}1 \\
497\end{array}$ & $\begin{array}{c}5 \% \text { naz. o } 3 \text { seggi } \\
\text { plurality }\end{array}$ \\
\hline Germania V & 2 & 672 & $\begin{array}{l}\text { MG } \\
\text { PR-naz. }\end{array}$ & $\begin{array}{l}\text { plurality } \\
\text { LR-Hare }\end{array}$ & $\begin{array}{l}328 \\
344\end{array}$ & $\begin{array}{l}48,8 \\
51,2\end{array}$ & $\begin{array}{r}328 \\
1\end{array}$ & $\begin{array}{c}1 \\
672\end{array}$ & $\begin{array}{c}5 \% \text { naz. o } 3 \text { seggi } \\
\text { plurality }\end{array}$ \\
\hline
\end{tabular}




\begin{tabular}{|c|c|c|c|c|c|c|c|c|c|}
\hline Nuova Zelanda & 2 & 120 & $\begin{array}{l}\text { MG } \\
\text { PR-naz. }\end{array}$ & $\begin{array}{l}\text { plurality } \\
\text { St. Laguë }\end{array}$ & $\begin{array}{r}653 \\
55\end{array}$ & $\begin{array}{l}54,2 \\
45,8\end{array}$ & $\begin{array}{r}65 \\
1\end{array}$ & $\begin{array}{r}1 \\
120\end{array}$ & $\begin{array}{l}5 \% \text { naz. o } 1 \text { seggio } \\
\text { plurality }\end{array}$ \\
\hline Venezuela & 2 & 203 & $\begin{array}{l}\text { MG } \\
\text { PR-reg. } \\
\text { PR-naz. }\end{array}$ & $\begin{array}{l}\text { plurality } \\
\text { d'Hondt } \\
\text { compensativi }\end{array}$ & $\begin{array}{r}100 \\
99 \\
4\end{array}$ & $\begin{array}{r}49,3 \\
48,8 \\
1,9\end{array}$ & $\begin{array}{r}100 \\
20 \\
1\end{array}$ & $\begin{array}{l}1 \\
9,9\end{array}$ & $\overline{1}$ quoziente \\
\hline $\begin{array}{l}\text { A semi-correzione } \\
\text { (con voto disgiunto): } \\
\text { Italia }\end{array}$ & 2 & 630 & $\begin{array}{l}\text { MG } \\
\text { PR-naz. }\end{array}$ & $\begin{array}{l}\text { plurality } \\
\text { LR-Hare }\end{array}$ & $\begin{array}{l}475 \\
155\end{array}$ & $\begin{array}{l}75,4 \\
24,6\end{array}$ & $\begin{array}{r}475 \\
1\end{array}$ & $\begin{array}{r}1 \\
155\end{array}$ & $4 \%$ naz. \\
\hline
\end{tabular}

Note:

1 Prima dell'applicazione della formula LR-Hare, i voti dei partiti che non hanno superato la soglia del $4 \%$ sono redistribuiti proporzionalmente a quei partiti che invece l'hanno superata.

2 Nel 1990 la soglia di sbarramento si applica separatamente nei due territori coincidenti con l'ex Germania occidentale e l'ex Germania orientale.

3 Di cui cinque seggi sono riservati alla minoranza Maori.

Fonti: Albania I: Inter-Parliamentary Union (Ipu); Germania I, II, III, e IV: Lijphart (1994), Kaase (1984); Germania V: American Institute for Contemporary German Studies (1996); Nuova Zelanda: Vowles (1995), Mackerras (1994); Venezuela: Ipu, Jones (1995); Italia: D’Alimonte e Chiaramonte (1993). 
Il sistema elettorale italiano introdotto nel 1993 è l'unico caso di semi-correzione pura. Come già in parte anticipato, si distingue dal tipo dei sistemi misti a correzione completa perché 1) la ripartizione proporzionale dei seggi viene effettuata non sulla base del totale dei seggi bensì sulla quota predeterminata per quel livello (non vi è pertanto una subordinazione gerarchica della parte maggioritaria rispetto a quella proporzionale), e 2) lo scorporo proporzionale avviene sui voti e non sui seggi. Si tratta comunque di un sistema a correzione, dato che le due arene competitive sono meccanicamente e territorialmente connesse.

Il funzionamento del sistema misto italiano è, in estrema sintesi, il seguente. In 475 collegi uninominali (pari al 75,4\% del totale dei seggi) vince il candidato che ottiene più voti, secondo la regola plurality. Questi era preventivamente, e obbligatoriamente, collegato ad una (o più di una) lista concorrente in sede proporzionale. A tale lista (o insieme di liste), posto che essa abbia superato la soglia di sbarramento del $4 \%$, viene sottratto un numero di voti pari a quello che è stato necessario alla vittoria del suo candidato di collegio - ossia il numero di voti (aumentato di una unità) ottenuti dal candidato secondo classificato in quello stesso collegio, e comunque non meno del $25 \%$ dei voti validi nel collegio (scorporo parziale dei voti) ${ }^{28}$. Lo scorporo dei voti si ripete ovviamente per tutti i collegi uninominali. La distribuzione dei 155 seggi proporzionali avviene allora non a partire dalla quota dei voti effettivamente ricevuti da ogni lista in questo comparto, bensì da quella stessa quota diminuita di tutti gli scorpori a carico della lista.

Si può ben capire, a questo punto, come i sistemi misti a semi-correzione tendano ad avvantaggiare $i$ concorrenti più forti in sede uninominale più di quanto facciano i sistemi misti a correzione completa: in primo luogo, la base di riferimento per l'assegnazione dei seggi proporzionali si riduce ad una quota nel caso italiano minoritaria - del totale dell'assemblea parlamentare; in secondo luogo, lo scorporo dei voti in luogo dello scorporo dei seggi lascia alle formazioni vincenti nel maggioritario (che in alcuni collegi saranno pur perdenti, eludendo in tal

28 Se più liste sono collegate al candidato vincente, la detrazione dei voti avviene in ragione proporzionale della loro forza elettorale nell'ambito del collegio. Per un approfondimento sul sistema elettorale italiano si rinvia a D'Alimonte e Chiaramonte (1993). 
caso i costi dello scorporo) una parte più consistente di voti da spendere a livello proporzionale. Sia dal punto di vista del modo in cui sono congegnati sia da quello degli effetti attesi, la distinzione tra sistemi a correzione completa e sistemi a semicorrezione appare quindi più che giustificata.

\section{Coesistenza e tipi ibridi}

Nella tabella 4 sono presentati i casi residui, che non configurano né il sistema a combinazione, né il sistema a correzione, almeno nella loro versione «pura». Tra questi casi ve ne sono due per così dire storici: il sistema danese del 1918 e quello francese del periodo 1951-56.

Quest'ultimo costituisce l'unico esempio del modello di coesistenza pura. All'interno del territorio nazionale convivono cioè due aree separate in una delle quali è applicata una formula maggioritaria (in questo caso il sistema maggioritario a doppio turno in collegi plurinominali) e nell'altra una formula proporzionale (per l'esattezza due distinte formule proporzionali nel caso francese: il quoziente naturale e i più alti resti per l'area di Parigi, il metodo d'Hondt nel resto del territorio) (Lijphart 1994).

Passando ai tipi ibridi, il sistema elettorale danese rappresenta un caso che contempla allo stesso tempo le modalità della coesistenza e della correzione. In sostanza, rispetto al sistema francese appena analizzato, oltre ai livelli proporzionale e maggioritario di seggi - territorialmente distinti - sono presenti due ulteriori livelli (uno provinciale e uno nazionale) concepiti a fini correttivi (o compensativi della distorsione realizzatasi) (Elklit 1992).

Nell'Ungheria post-comunista, a prescindere dalla modifica attuata con l'aumento della soglia legale di sbarramento tra il 1990 e il 1994, il sistema a semi-correzione già visto per l'Italia convive con il sistema a combinazione. Innanzitutto, nei 176 collegi uninominali in cui è diviso il territorio nazionale viene adottata la formula majority-plurality (doppio turno aperto). Per quanto riguarda la ripartizione proporzionale dei seggi, poi, essa avviene a due livelli, regionale e nazionale. In sede regionale vengono assegnati al massimo 152 seggi sulla base dei quozienti interi calcolati col il metodo Droop. Fin qui il sistema elettorale misto ungherese sarebbe solo a combinazione. C'è 
TАB. 4. Caratteristiche principali di altri quattro sistemi elettorali misti, a coesistenza e ibridi

\begin{tabular}{|c|c|c|c|c|c|c|c|c|c|}
\hline $\begin{array}{l}\text { Sistema } \\
\text { Elettorale }\end{array}$ & $\begin{array}{l}\text { Numero } \\
\text { di voti }\end{array}$ & $\begin{array}{l}\text { Ampiezza } \\
\text { assemblea } \\
\text { parlament. }\end{array}$ & $\begin{array}{l}\text { Livelli di } \\
\text { ripartizione } \\
\text { dei seggi } 1\end{array}$ & $\begin{array}{l}\text { Formula } \\
\text { elettorale }\end{array}$ & $\begin{array}{l}\text { Numero } \\
\text { medio } \\
\text { di seggi }\end{array}$ & $\begin{array}{l}\% \text { di seggi } \\
\text { per ciascun } \\
\text { livello }\end{array}$ & $\begin{array}{l}\text { Numero } \\
\text { medio } \\
\text { collegi }\end{array}$ & $\begin{array}{c}\text { Dimensione } \\
\text { media } \\
\text { dei collegi }\end{array}$ & $\begin{array}{c}\text { Soglie } \\
\text { legali }\end{array}$ \\
\hline \multicolumn{10}{|l|}{ Sistemi a coesistenza: } \\
\hline \multirow[t]{3}{*}{ Francia } & 1 & 544 & MG-altro & majority & 116 & 21,3 & 25,5 & 4,8 & \\
\hline & & & PR-Parigi & LR-Hare & 75 & 13,8 & 8 & 9,4 & - \\
\hline & & & PR-altro & d'Hondt & 353 & 64,9 & 69,5 & 5,1 & - \\
\hline \multicolumn{10}{|l|}{ Tipi ibridi: } \\
\hline \multirow[t]{4}{*}{ Danimarca } & 1 & 140 & MG-altro & plurality & 93 & 66,4 & 93 & 1 & \\
\hline & & & PR-Copenhagen (I) & d'Hondt & 24 & 17,1 & 1 & 24 & - \\
\hline & & & PR-altro & LR-Hare & $20^{2}$ & 14,3 & 2 & 56,5 & - \\
\hline & & & PR-naz. & LR-Hare & 33 & 2,2 & 1 & & $\begin{array}{l}1 \text { seggio } M G \\
\text { o } 1 \text { seggio } P R(I)\end{array}$ \\
\hline \multirow[t]{3}{*}{ Ungheria I } & 2 & 386 & MG & majority-plurality & 176 & 45,6 & 176 & 1 & \\
\hline & & & PR-reg. & Droop & 120 & 31,1 & 20 & 6,0 & $4 \%$ naz. \\
\hline & & & PR-naz. & LR-Hare & 90 & 23,3 & 1 & 90 & $4 \%$ naz. \\
\hline \multirow[t]{3}{*}{ Ungheria II } & 2 & 386 & MG & majority-plurality & 176 & 45,6 & 176 & 1 & \\
\hline & & & PR-reg. & Droop & 125 & 32,4 & 20 & 6,0 & $5 \%$ naz. \\
\hline & & & PR-naz. & LR-Hare & 85 & 22,0 & 1 & 85 & $5 \%$ naz. \\
\hline
\end{tabular}

Note:

1 I livelli di (semi-)correzione sono in corsivo.

2 Seggi compensativi provinciali atti ad assicurare la proporzionalità dell'esito nelle due province che adottano il plurality in collegi uninominali.

3 Seggi compensativi nazionali atti ad assicurare la proporzionalità complessiva dell'esito.

Fonti: Francia: Lijphart (1994); Danimarca: Elklit (1992); Ungheria I: Gabel (1995), Kukorelli (1991); Ungheria II: Ipu, Rady (1994). 
però anche un livello nazionale, nel quale i 58 seggi minimi previsti più i seggi rimasti non assegnati nelle regioni vengono distribuiti alle liste sulla base dei rispettivi surplus di voti. Poiché costituiscono il surplus di voti non solo i voti-resto delle liste regionali che non sono stati utili per ricevere seggi ma anche $\mathrm{i}$ voti dei candidati perdenti di collegio calcolati sull'esito del primo turno di votazione (Gabel 1995), questa parte del sistema elettorale configura il modello a semi-correzione ${ }^{29}$.

Esaurita la descrizione delle caratteristiche principali dei sistemi elettorali misti, non resta che passare all'analisi del loro impatto sui rispettivi contesti di applicazione, in modo da valutare le differenze che si registrano e compiere poi alcune considerazioni conclusive.

\section{Gli effetti sul sistema partitico: una prima valutazione}

Le variabili che consentono di apprezzare, nell'ambito di una comparazione ad ampio raggio, similarità e differenze nell'impatto dei sistemi elettorali misti sui sistemi partitici sono quelle classiche della deviazione dalla proporzionalità - qui utilizzeremo l'indice D di Loosemore-Hanby (1971) -, del numero effettivo di partiti elettorali $\left(\mathrm{Neff}_{\mathrm{e}}\right)$ e parlamentari ( $\mathrm{Neff}_{\mathrm{p}}$ ) (Laasko e Taagepera 1979), della riduzione relativa del numero dei partiti appunto dall'arena elettorale all'arena parlamentare $\left(\mathrm{r}=\left(\mathrm{Neff}_{\mathrm{e}}-\mathrm{Neff}_{\mathrm{p}}\right) / \mathrm{Neff}_{\mathrm{e}}\right)$, nonché la frequenza di maggioranze parlamentari monopartitiche e la frequenza di maggioranze «fabbricate» dal sistema elettorale (ovviamente, attraverso gli effetti distorsivi da questo prodotti).

La tabella 5 illustra i valori medi degli indici appena elencati per ogni caso in esame. Ciò che emerge a prima vista è l'estrema variabilità dei dati. La disproporzionalità assume il valore minimo per la Danimarca $(4,46)$ e raggiunge il massimo in riferimento al sistema elettorale Georgia II $(53,78)$. Il numero effettivo di partiti elettorali varia tra 2,20 (Albania I) e 12,83 (ancora Georgia II), mentre il numero effettivo di partiti parlamentari tra 1,31 (Albania II) e 10,35 (Russia). Parallelamente,

29 Qui, diversamente dal sistema elettorale italiano per la Camera dei deputati, la semi-correzione si fonda sullo scorporo totale e non parziale dei voti maggioritari. Alle liste collegate ai candidati di collegio vincenti vengono cioè detratti tutti $i$ voti ottenuti da quei candidati e non una quota di essi. 
TAB. 5. Disproporzionalità e impatto sul sistema partitico dei 24 sistemi elettorali misti suddivisi per tipi

\begin{tabular}{|c|c|c|c|c|c|c|}
\hline $\begin{array}{l}\text { Sistema } \\
\text { elettorale }\end{array}$ & $\mathrm{D}$ & $\mathrm{Neff}_{\mathrm{e}}$ & $\mathrm{Neff}_{\mathrm{p}}$ & $\mathrm{r}$ & $\begin{array}{l}\text { Magg. } \\
\text { parlam. }\end{array}$ & $\begin{array}{l}\text { Magg } \\
\text { fabbr. }\end{array}$ \\
\hline \multicolumn{7}{|c|}{ Combinazione con voto fuso: } \\
\hline Albania II & 31,61 & 2,80 & 1,31 & 0,53 & $1 / 1$ & $0 / 1$ \\
\hline Corea (Sud) & 13,63 & 4,51 & 3,16 & 0,30 & $0 / 1$ & $0 / 1$ \\
\hline Taiwan & 5,21 & 2,82 & 2,46 & 0,13 & $1 / 1$ & $1 / 1$ \\
\hline \multicolumn{7}{|c|}{ Combinazione con voto disgiunto: } \\
\hline Bulgaria & 6,80 & 2,75 & 2,42 & 0,12 & $1 / 1$ & $1 / 1$ \\
\hline Croazia & 15,36 & 3,61 & 2,32 & 0,36 & $1 / 1$ & $1 / 1$ \\
\hline Georgia I & n.d. & n.d. & n.d. & n.d. & n.d. & n.d. \\
\hline Georgia II & 53,78 & 12,83 & 3,51 & 0,73 & $0 / 1$ & $0 / 1$ \\
\hline Giappone & 20,17 & 4,29 & 2,94 & 0,31 & $0 / 1$ & $0 / 1$ \\
\hline Lituania I & 10,62 & 3,68 & 3,04 & 0,17 & $1 / 1$ & $1 / 1$ \\
\hline Lituania II & 27,75 & 7,64 & 3,32 & 0,57 & $1 / 1$ & $1 / 1$ \\
\hline Messico & 9,82 & 2,83 & 2,29 & 0,19 & $1 / 1$ & $0 / 1$ \\
\hline Russia & 33,21 & 9,68 & 10,35 & $-0,16$ & $0 / 2$ & $0 / 2$ \\
\hline \multicolumn{7}{|c|}{ Correzione con voto fuso: } \\
\hline Albania I & 5,67 & 2,20 & 1,97 & 0,10 & $1 / 1$ & $0 / 1$ \\
\hline Germania I & 8,40 & 5,71 & 4,65 & 0,19 & $0 / 1$ & $0 / 1$ \\
\hline \multicolumn{7}{|c|}{ Correzione con voto disgiunto: } \\
\hline Germania II & 7,21 & 4,21 & 3,63 & 0,14 & $0 / 1$ & $0 / 1$ \\
\hline Germania III & 3,31 & 3,17 & 2,95 & 0,07 & $0 / 8$ & $0 / 8$ \\
\hline Germania IV & 1,35 & 3,56 & 3,47 & 0,03 & $0 / 1$ & $0 / 1$ \\
\hline Germania V & 5,85 & 3,16 & 2,78 & 0,12 & $0 / 2$ & $0 / 2$ \\
\hline Nuova Zelanda & 7,35 & 4,35 & 3,76 & 0,14 & $0 / 1$ & $0 / 1$ \\
\hline Venezuela & 9,73 & 5,43 & 4,50 & 0,17 & $0 / 1$ & $0 / 1$ \\
\hline \multicolumn{7}{|c|}{$\begin{array}{l}\text { Semi-correzione } \\
\quad(\text { con voto disgiunto): }\end{array}$} \\
\hline Italia & 14,40 & 7,35 & 6,11 & 0,17 & $0 / 2$ & $0 / 2$ \\
\hline \multicolumn{7}{|l|}{ Coesistenza: } \\
\hline Francia & 8,92 & 5,76 & 5,83 & $-0,02$ & $0 / 2$ & $0 / 2$ \\
\hline \multicolumn{7}{|l|}{ Tipi ibridi: } \\
\hline Danimarca & 4,46 & 4,08 & 3,89 & 0,05 & $0 / 1$ & $0 / 1$ \\
\hline Ungheria I & 22,82 & 6,75 & 3,78 & 0,44 & $0 / 1$ & $0 / 1$ \\
\hline Ungheria II & 21,19 & 5,51 & 2,89 & 0,47 & $1 / 1$ & $1 / 1$ \\
\hline
\end{tabular}

Legenda: $\mathrm{D}$ indice di Loosemore-Hanby; Neff $_{\mathrm{e}}$ numero effettivo di partiti elettorali; Neff ${ }_{p}$ numero effettivo di partiti parlamentari; $r$ riduzione relativa del numero effettivo di partiti; Magg. parlam. frequenza di maggioranze parlamentari monopartitiche; Magg. fabbr. frequenza di maggioranze parlamentari monopartitiche «fabbricate»; n.d. dato non disponibile. 


\section{TAB. 5. (segue)}

Fonti: i valori degli indici per Germania I, II, III e IV e per la Francia sono tratti da Lijphart (1994), mentre quelli della Danimarca da Elklit (1992); gli altri sono una mia elaborazione su dati elettorali ricavati dalle seguenti fonti: Albania II: Inter-Parliamentary Union (Ipu), AA.VV. (1994); Corea (Sud): Morriss (1996); Taiwan: Hsieh e Niou (1996); Bulgaria: Koulov (1995); Croazia: dati ufficiali, Kasapovic (1996); Georgia II: Ipu, Allison (1996); Giappone: Ipu; Lituania I: Ipu; Lituania II: dati ufficiali (Seimas); Messico: dati ufficiali (Instituto Federal Electoral), Ipu; Russia: dati ufficiali (TsIK) sia per le elezioni del 1993 che per quelle del 1995; Albania I: Szajkowski (1994); Germania V: American Institute for Contemporary German Studies (1996); Nuova Zelanda: dati ufficiali; Venezuela: dati ufficiali (Consejo Supremo Electoral), Landman (1995); Ungheria I: Martis, Z. Kovacs, D. Kovacs e S. Peter (1992); Ungheria II: Fitzmaurice (1995), Rady (1994); Italia: dati ufficiali (Camera dei deputati).

anche l'indice $r$ presenta valori molto diversi tra loro: è massimo per Georgia II $(0,73)$ e minimo - anzi addirittura negativo, a causa dell'elezione di un alto numero di indipendenti - per Francia $(-0,02)$ e ancor più per Russia $(-0,16)$. Maggioranze parlamentari monopartitiche si verificano in 9 casi su $24^{30}$, ed in sei di essi si tratta di maggioranze fabbricate.

L'estrema variabilità di cui si è detto è confermata - come mostra la tabella 6 (ultima riga) - dagli alti valori del coefficiente di variazione calcolato su tutti i nostri 24 sistemi elettorali misti. Non si tratta però di un qualcosa di inaspettato, né lo è la circostanza che non si manifesti una qualche relazione tra le variabili in questione e la quota relativa di seggi maggioritari di ciascun caso: come più volte ripetuto, infatti, la loro complessa architettura rende questi sistemi (e il loro impatto, soprattutto se misurato solo attraverso gli indici di disproporzionalità e di frammentazione) molto diversi l'uno dall'altro, e non soltanto (o primariamente) per via del differente «dosaggio» tra seggi maggioritari e seggi proporzionali.

A questo punto è interessante osservare se la variabilità si riduce distinguendo tra $\mathrm{i}$ tipi e i sotto-tipi individuati nelle sezioni precedenti - tenendo comunque conto che la significatività statistica del dato diminuisce al diminuire dei casi presi in esame. Questo, stando ancora ai valori del coefficiente di variazione rilevati, accade solo in parte. Certamente la variabilità interna agli effetti prodotti dai sistemi misti a correzione completa si riduce notevolmente, anche se bisogna ammettere che su

30 I casi oggetto della nostra analisi si sono ridotti da 25 a 24 a causa dell'indisponibilità di dati completi per Georgia I. 
TAB. 6. Disproporzionalità e impatto sul sistema partitico a seconda dei tipi di sistema elettorale misto

\begin{tabular}{|c|c|c|c|c|c|c|c|c|}
\hline $\begin{array}{l}\text { Tipo di } \\
\text { sistema misto }\end{array}$ & $\begin{array}{l}\mathrm{N} \\
\text { casi }\end{array}$ & $\mathrm{M} / \mathrm{Cv}$ & $\mathrm{D}$ & $\mathrm{Neff}_{\mathrm{e}}$ & $\operatorname{Neff}_{p}$ & r & $\begin{array}{l}\text { Magg. } \\
\text { parlam. }\end{array}$ & $\begin{array}{l}\text { Magg. } \\
\text { fabbr. }\end{array}$ \\
\hline $\begin{array}{l}\text { Combinazione } \\
\text { con voto fuso }\end{array}$ & 3 & $\begin{array}{l}\mathrm{M} \\
\mathrm{Cv}\end{array}$ & $\begin{array}{r}16,82 \\
0,80\end{array}$ & $\begin{array}{l}3,38 \\
0,29\end{array}$ & $\begin{array}{l}2,31 \\
0,41\end{array}$ & $\begin{array}{l}0,32 \\
0,63\end{array}$ & 0,67 & 0,33 \\
\hline $\begin{array}{l}\text { Combinazione } \\
\text { con voto disgiunto }\end{array}$ & 8 & $\begin{array}{l}\mathrm{M} \\
\mathrm{Cv}\end{array}$ & $\begin{array}{r}22,19 \\
0,71\end{array}$ & $\begin{array}{l}5,91 \\
0,63\end{array}$ & $\begin{array}{l}3,77 \\
0,71\end{array}$ & $\begin{array}{l}0,29 \\
0,96\end{array}$ & 0,56 & 0,44 \\
\hline - Totale combinazione & e 11 & $\begin{array}{l}\mathrm{M} \\
\mathrm{Cv}\end{array}$ & $\begin{array}{r}20,81 \\
0,67\end{array}$ & $\begin{array}{l}5,46 \\
0,62\end{array}$ & $\begin{array}{l}3,48 \\
0,69\end{array}$ & $\begin{array}{l}0,31 \\
0,81\end{array}$ & 0,58 & 0,42 \\
\hline $\begin{array}{l}\text { Correzione comp. } \\
\text { con voto fuso }\end{array}$ & 2 & $\begin{array}{l}\mathrm{M} \\
\mathrm{Cv}\end{array}$ & $\begin{array}{l}7,03 \\
0,27\end{array}$ & $\begin{array}{l}3,95 \\
0,63\end{array}$ & $\begin{array}{l}3,31 \\
0,57\end{array}$ & $\begin{array}{l}0,14 \\
0,40\end{array}$ & 0,50 & 0,00 \\
\hline $\begin{array}{l}\text { Correzione comp. } \\
\text { con voto disgiunto }\end{array}$ & 6 & $\begin{array}{l}\mathrm{M} \\
\mathrm{Cv}\end{array}$ & $\begin{array}{l}5,80 \\
0,52\end{array}$ & $\begin{array}{l}3,98 \\
0,22\end{array}$ & $\begin{array}{l}3,52 \\
0,18\end{array}$ & $\begin{array}{l}0,11 \\
0,48\end{array}$ & 0,00 & 0,00 \\
\hline $\begin{array}{l}\text { - Totale correzione } \\
\text { completa }\end{array}$ & 8 & $\begin{array}{l}\mathrm{M} \\
\mathrm{Cv}\end{array}$ & $\begin{array}{l}6,11 \\
0,45\end{array}$ & $\begin{array}{l}3,97 \\
0,30\end{array}$ & $\begin{array}{l}3,46 \\
0,26\end{array}$ & $\begin{array}{l}0,12 \\
0,44\end{array}$ & 0,06 & 0,00 \\
\hline Semi-correzione (Italia & a) 1 & & 14,40 & 7,35 & 6,11 & 0,17 & 0,00 & 0,00 \\
\hline - Totale correzione & 9 & $\begin{array}{l}\mathrm{M} \\
\mathrm{Cv}\end{array}$ & $\begin{array}{l}7,03 \\
0,53\end{array}$ & $\begin{array}{l}4,35 \\
0,36\end{array}$ & $\begin{array}{l}3,76 \\
0,32\end{array}$ & $\begin{array}{l}0,12 \\
0,42\end{array}$ & 0,05 & 0,00 \\
\hline Coesistenza (Francia) & 1 & & 8,92 & 5,76 & 5,83 & $-0,02$ & 0,00 & 0,00 \\
\hline Danimarca & 1 & & 4,46 & 4,08 & 3,89 & 0,05 & 0,00 & 0,00 \\
\hline Ungheria I+II & 2 & $\begin{array}{l}\mathrm{M} \\
\mathrm{Cv}\end{array}$ & $\begin{array}{r}22,01 \\
0,05\end{array}$ & $\begin{array}{l}6,13 \\
0,14\end{array}$ & $\begin{array}{l}3,34 \\
0,18\end{array}$ & $\begin{array}{l}0,46 \\
0,04\end{array}$ & 0,50 & 0,50 \\
\hline $\begin{aligned}- & \text { Totale tutti } \\
& i \text { sistemi misti }\end{aligned}$ & 24 & $\begin{array}{l}\mathrm{M} \\
\mathrm{Cv}\end{array}$ & $\begin{array}{r}14,53 \\
0,84\end{array}$ & $\begin{array}{l}4,94 \\
0,50\end{array}$ & $\begin{array}{l}3,64 \\
0,50\end{array}$ & $\begin{array}{l}0,22 \\
0,93\end{array}$ & 0,26 & 0,17 \\
\hline
\end{tabular}

Legenda: $\mathrm{M}$ media; $\mathrm{Cv}$ coefficiente di variazione; $\mathrm{D}$ indice di Loosemore-Hanby; $\mathrm{Neff}_{\mathrm{e}}$ numero effettivo di partiti elettorali; Neff $\mathrm{p}$ numero effettivo di partiti parlamentari; $r$ riduzione relativa del numero effettivo di partiti; Magg. parlam. frequenza di maggioranze parlamentari monopartitiche; Magg. fabbr. frequenza di maggioranze parlamentari monopartitiche «fabbricate».

Fonti: elaborazione propria su fonti di cui a tab. 5 . 
questa categoria pesano molto i cinque casi riconducibili alla sola Germania. Quanto ai sistemi a combinazione essa rimane alta, a testimonianza sì di una forte diversità nell'impatto di questi sistemi elettorali, ma anche a causa dell'incidenza di casi che, come vedremo meglio tra breve, possono essere senz'altro definiti come devianti.

Tanta variazione nell'influenza dei sistemi misti, pur distinguendo quest'ultimi in gruppi più ristretti, necessita di essere valutata attentamente, anche perché potrebbe dar adito ad alcune perplessità circa la classificazione cui siamo pervenuti. Essa sembra derivare da tre ordini di motivi, tra loro connessi e complementari.

Innanzitutto, gli indici qui utilizzati - e del resto ampiamente diffusi in letteratura - sono talvolta di difficile applicazione rispetto ad elezioni svoltesi con sistemi elettorali misti, specie se la struttura della competizione tra partiti/candidati è fortemente diversificata tra parte proporzionale e parte maggioritaria (in sostanza, l'espressione sintetica del valore di un indice richiede in taluni casi delle assunzioni, ossia delle scelte che implicano margini di arbitrarietà). $\mathrm{Ne}$ consegue che certi dati rischiano di sovra/sotto-dimensionare un fenomeno e che l'accuratezza della comparazione può presentare difficoltà supplementari a quelle che solitamente si rinvengono. Ad ogni modo questo è più che altro un avvertimento e una raccomandazione ad interpretare le relazioni ipotetiche in base alle linee di tendenza che esprimono piuttosto che nei termini della precisione del dato numerico.

In secondo luogo, molti dei sistemi elettorali qui esaminati hanno avuto un'applicazione limitata ad una sola elezione e non sono quindi analizzati «a regime». $\grave{E}$ noto, infatti, che la riforma dei sistemi elettorali, tanto più quanto maggiore è la portata dei cambiamenti introdotti, esplica i suoi effetti nell'arco di almeno due-tre elezioni, per via del necessario processo di apprendimento delle nuove regole del gioco da parte di elettori e partiti. Inoltre, una buona parte dei nostri casi fa riferimento a democrazie in transizione, il che aggrava ancor più il problema sollevato immediatamente sopra. In tali circostanze si riscontra una partecipazione spesso «disordinata» di miriadi di liste e di candidati alla quale si accompagna una forte dispersione del voto degli elettori, e ciò non si spiega soltanto con l'inevitabile costo per l'apprendimento bensì ha a che fare con le dinamiche proprie dell'instaurazione di un nuovo regime. Senza scendere nel dettaglio di molti tra i nostri casi, basti fare l'esempio di Geor- 
gia II (elezioni del 1995). Qui l'effetto congiunto del sistema elettorale (soprattutto della soglia di sbarramento) e della mancanza di coordinamento strategico di partiti ed elettori (la frammentazione partitica in entrata è $\mathrm{Neff}_{\mathrm{e}}=12,83$, un valore eccezionalmente alto) ha prodotto una serie di conseguenze pressoché irripetibili: 1) oltre il $60 \%$ dei voti proporzionali è andato sprecato (è stato cioè attribuito a partiti sotto-soglia), per cui 2) la riduzione del numero dei partiti è stata elevatissima $(r=$ $0,73)$, e 3) così pure la disproporzionalità $(D=53,78)$ e 4) un partito con appena il $23,7 \%$ dei voti ha sfiorato da solo la maggioranza assoluta dei seggi in parlamento. Insomma, è lecito aspettarsi che l'applicazione ripetuta di questi sistemi elettorali, soprattutto in certi contesti, porti ad una progressiva stabilizzazione dei comportamenti di elettori e partiti e con essa ad una minore variazione tra $\mathrm{i}$ casi in esame.

In terzo luogo, e questa è certamente la considerazione più importante, si deve rilevare che esiste una componente per così dire strutturale nella variazione d'impatto interna ai tipi principali dei nostri sistemi elettorali misti, soprattutto al tipo a combinazione. Essa discende dai livelli differenti di quello che abbiamo chiamato il «dosaggio» tra seggi maggioritari e seggi proporzionali, nonché dall'entità della soglia di accesso alla rappresentanza e dalla dimensione media dei collegi - il che si riflette inevitabilmente sugli effetti distorsivi e selettivi prodotti. Del resto, ciò non rappresenta affatto un elemento di debolezza della distinzione tra $\mathrm{i}$ tipi a combinazione, a correzione e a coesistenza, poiché il criterio che soggiace a tale distinzione - la modalità di raccordo tra i livelli maggioritario e proporzionale di assegnazione dei seggi - viene prima, ed è logicamente più rilevante, delle variabili in questione. L'alternativa di differenziare i sistemi misti in base, diciamo, alla percentuale di seggi maggioritari (o proporzionali) che prevedono sarebbe infatti un rimedio peggiore del (presunto) male: metterebbe insieme ad esempio Russia (o Lituania) e Germania, ossia casi in realtà distanti tra loro tanto in riferimento agli elementi costitutivi quanto per gli esiti che generano.

A prescindere dalle doverose considerazioni appena sviluppate, resta il fatto che è comunque possibile trarre dai dati delle tabelle 5 e 6 indicazioni corroboranti la bontà della classificazione dei sistemi elettorali misti qui presentata, al di là della circostanza fondamentale che essa sia stata costruita a partire dagli input legali. In particolare, ne escono confermate due ipotesi 
alle quali si era già accennato in sede di descrizione dei tipi di sistemi elettorali misti: quella di una tendenziale maggiore capacità manipolativa dei sistemi a combinazione rispetto ai sistemi a correzione e quella di una sostanziale diversità d'impatto tra sistemi a correzione completa e sistemi a semi-correzione (puri o ibridi). La validità della prima ipotesi è testimoniata soprattutto dai valori medi della disproporzionalità $(20,81$ nei sistemi a combinazione, 7,03 nei sistemi a correzione) e della riduzione relativa del numero di partiti (rispettivamente 0,31 e 0,12 ), nonché della frequenza di maggioranze parlamentari monopartitiche $(0,58$ contro 0,05$)$ e di maggioranze fabbricate $(0,42$ contro $0)$. La seconda ipotesi trova riscontro nell'immediato confronto degli stessi dati tra il gruppo dei sistemi a correzione completa e i tre casi a semi-correzione: Italia (puro), e Ungheria I e II (ibridi).

Per ricapitolare, la sintesi tra i due principi di rappresentanza a cui ciascun sistema elettorale misto è pervenuto presenta un notevole grado di diversità che si riflette in altrettanto diverse capacità di compensazione proporzionalistica o, inversamente, di delineare maggioranze. Cosa peraltro che era lecito attendersi anche alla luce della difformità dei contesti di applicazione - si pensi, ad esempio, alla distanza esistente tra Nuova Zelanda ed Albania. Tuttavia, e con la cautela imposta da quanto appena detto, emerge una significativa distinzione anche sul versante degli output elettorali tra i sistemi misti a combinazione e quelli a correzione e, all'interno di quest'ultimo tipo, tra i sistemi a correzione completa e quelli a semi-correzione - il che rappresenta un'ulteriore conferma alla validità della nostra classificazione dei sistemi misti.

\section{Conclusioni: «il meglio dei due mondi»?}

La recente diffusione dei sistemi elettorali misti sembra rispondere ad una logica di compromesso «il cui effetto complessivo è quello di erodere la distintività del modello storico di ciascun paese» (Dunleavy e Margetts 1995). La loro scelta rappresenta il tentativo esplicito o implicito di compendiare «il meglio dei due mondi», ovvero dei principi maggioritario e proporzionale di rappresentanza. $\mathrm{Ma}$ i sistemi elettorali misti configurano davvero «il meglio dei due mondi»?

L'analisi che abbiamo qui condotto, incentrata particolar- 
mente sugli aspetti descrittivi, costituisce solo un primo tentativo di approfondimento del tema. Una risposta definitiva al quesito ora sollevato è dunque allo stato impossibile. Sulla scorta delle informazioni acquisite si possono tuttavia avanzare alcune considerazioni che potranno tornare utili in future ricerche.

Innanzitutto, abbiamo rilevato che la notevole variabilità tanto delle caratteristiche dei sistemi elettorali misti quanto degli effetti da questi prodotti sul sistema partitico, considerato soprattutto che molti hanno avuto un'applicazione limitata, non rende al momento agevole trattarli in maniera univoca e compararli con altri tipi di sistemi elettorali vigenti da lungo tempo. Questo è uno dei motivi per cui, confrontando i dati medi delle variabili di impatto dei sistemi elettorali misti (e dei suoi tipi) con gli stessi dei sistemi elettorali proporzionali e dei sistemi elettorali maggioritari (per i quali si rimanda a Lijphart 1994), non sembra emergere una «centralità» dei primi rispetto ai secondi. Ad ogni buon conto sarebbe scorretto fondare la legittimità del trattamento dei sistemi misti come categoria autonoma sulla premessa che essi devono necessariamente essere generatori di effetti - quelli prima investigati - per così dire mediani rispetto ai tipi polari maggioritario e proporzionale. Vediamo meglio perché.

In primo luogo, è doveroso ribadire che gli effetti ai quali ci stiamo riferendo - in particolare, disproporzionalità e numero dei partiti - sono operazionalizzati mediante variabili di tipo continuo: se già i soli sistemi maggioritari e proporzionali tendono a esprimere un ampio spettro dei valori che quelle variabili possono assumere, in più di un caso poi accavallandosi $\mathrm{e}$ mischiandosi tra loro, come è allora possibile attendersi dai sistemi misti che $\mathrm{i}$ valori ad essi associati si collochino a metà strada rispetto ai primi? Per fare un esempio, un sistema misto a combinazione potrebbe produrre effetti selettivi e distorsivi non dissimili da quelli di un sistema proporzionale che presenta un'elevata soglia di sbarramento, ma anche da quelli di un sistema maggioritario applicato in un contesto con forti differenziazioni regionali di voto.

In secondo luogo, considerare sul versante dell'output soltanto il formato del sistema partitico e la disproporzionalità è certamente riduttivo - ossia non esaurisce quanto implicato dal concetto di principio di rappresentanza e, quindi, nel nostro caso, dalla natura mista dei sistemi elettorali in questione. Così facendo, si trascurano altri aspetti importanti tra cui, solo per 
citarne alcuni, 1) le strategie di competizione dei partiti (coalizioni, apparentamenti, ecc.), 2) le modalità di selezione (accentrata/decentralizzata) e il tipo (lista/individuale) delle candidature 3) il grado di penetrazione del partito nel rapporto fra $\mathrm{i}$ candidati e gli elettori del loro collegio (o della loro circoscrizione), 4) gli incentivi strategici rispetto al voto.

$\mathrm{Si}$ può allora ben capire come l'interrogativo se i sistemi elettorali misti costituiscano la sintesi migliore di proporzionale e maggioritario non possa trovare una risposta solo confrontando l'entità della distorsione e il numero dei partiti, bensì richieda ulteriori approfondimenti.

A tal proposito, il livello di astrazione dell'analisi può avere un'influenza determinante sul tipo di risultati cui è possibile pervenire. Nonostante il fatto che $\mathrm{i}$ contributi ritenuti più importanti per la conoscenza del funzionamento dei sistemi elettorali siano venuti, negli ultimi dieci anni circa, da lavori fondati su una comparazione statistica rigorosa e sistematica eseguita su un gran numero di casi (Taagepera e Shugart 1989 e Lijphart 1994 sono solo i più significativi), nello stesso periodo si è assistito anche ad un rifiorire di analisi incentrate su singoli sistemi elettorali o su un numero ristretto di sistemi elettorali. Si è in sostanza considerato utile e in certa misura necessario ricondurre l'analisi dei sistemi elettorali - e le conoscenze acquisite anche grazie alle comparazioni ad ampio raggio - entro ambiti più circoscritti in modo da tener conto di ulteriori variabili (tra cui alcune specificamente nazionali) che interagiscono rispetto alla relazione tra sistemi elettorali e sistemi partitici. Rose, ad esempio, ha rilevato che «per comprendere il funzionamento dei sistemi elettorali in pratica, si devono considerare sia le proprietà generali di un sistema che lo specifico contesto nazionale. [...] Il funzionamento di un sistema elettorale non può essere infatti compreso semplicemente nei termini di categorie definitorie ed astratte; deve invece essere valutato mediante un'analisi del sistema-elettorale-nel-sistema-politico» $(1984,78)$. Questo sembra particolarmente appropriato per i sistemi elettorali misti. È solo scendendo nel livello di astrazione dell'analisi - e, conseguentemente, riducendo il numero di casi presi in esame - che è possibile infatti tener conto della complessità degli effetti da essi esercitati. Non a caso nei lavori basati su comparazioni variableoriented i cosiddetti effetti psicologici (Duverger 1951) sono stati pressoché negletti. In altre parole, l'obbiettivo di ricerca relativo al concreto e diverso operare dei sistemi elettorali misti 
impone di verificare i loro effetti sui rispettivi sistemi partitici tenendo conto, per dirla con Elster, dei «meccanismi causali», ossia della complessa mappa di rapporti tra il sistema elettorale e gli attori che, a vario titolo, concorrono a determinare la rappresentanza politica: gli elettori, i partiti, i candidati.

La peculiarità dei sistemi elettorali misti, ossia la combinazione di due livelli di ripartizione dei seggi governati rispettivamente da una formula proporzionale e da una formula maggioritaria, invita poi ad analisi più approfondite che siano in grado di «scomporre» la misurazione di variabili quali la disproporzionalità e il numero effettivo di partiti appunto in base a ciascuno di quei due livelli. Uno dei risultati più interessanti che può derivarne, soprattutto in riferimento ai sistemi misti che connettono l'arena proporzionale e quella maggioritaria sulla base del principio della correzione, è l'effettiva capacità di compensazione che la ripartizione proporzionale dei seggi ha nei confronti della distorsione realizzatasi a livello maggioritario. La raccolta dei dati elettorali nazionali dovrebbe quindi essere compiuta con riferimento alla diversa distribuzione dei voti e dei seggi che si attua in sede proporzionale e in sede maggioritaria.

Si tenga inoltre conto che, per quanto riguarda la maggior parte dei sistemi elettorali misti qui considerati, una loro analisi più approfondita potrebbe configurarsi anche come caso cruciale, nel senso che alcune delle variabili in questione - si pensi al voto diviso (ed eventualmente strategico) e alle possibilità della sua rilevazione insita nella disposizione del doppio voto si presentano in una forma insolita di cui beneficia la ricerca (Shugart 1985). Questa circostanza offre, infatti, l'opportunità rara di operare un controllo sulle preferenze degli elettori e sulle strategie dei partiti (Gabel 1995).

E solo proseguendo lungo queste direttrici di ricerca che saremo dunque in grado di rispondere compiutamente al quesito presente nel titolo di queste conclusioni.

\section{Riferimenti bibliografici}

AA.VV. (1994), Eastern Europe and the Commonwealth of Independent States 1994, $2^{\mathrm{a}}$ ed., London, Europa Publication Limited.

Allison, L. (1996), The Georgian Elections of November 1995, in «Electoral Studies», XV, pp. 275-280. 
Allison, L., A. Kukhianidze e M. Matsaberidze (1993), The Georgian Election of 1992, in «Electoral Studies», XII, pp. 174-179.

American Institute for Contemporary German Studies, The Johns Hopkins University (1996), Super Election Year 1994 Reports.

Balinski, M. e V. Ramirez Gonzalez (1996), A Case Study of Electoral Manipulation: The Mexican Laws of 1989 and 1994, in «Electoral Studies», XV, pp. 203-217.

Batt, J. (1990), The Hungarian General Election, in «Representation», XXIX, n. 107, pp. 7-11.

Blais, A. (1988), The Classification of Electoral Systems, in «European Journal of Political Research», XVIII, pp. 99-110.

- (1991) The Debate over Electoral Systems, in «International Political Science Review», XII, pp. 239-260.

Blais, A. e L. Massicotte (1996), Electoral Systems, in L. LeDuc, R.G. Niemi e P. Norris (a cura di), Comparing Democracies. Elections and Voting in Global Perspective, London, Sage, pp. 49-81.

Boston, J. (1987), Electoral Reform in New Zealand: The Report of The Royal Commision, in «Electoral Studies», VI, pp. 105-114.

Carducci, M. (1994), Sistemi elettorali misti e rappresentanza, in O. Massari e G. Pasquino (a cura di), Rappresentare e governare, Bologna, Il Mulino, pp. 181-219.

Cole, P. (1995), Bonus Seats in the German Electoral System, in «Representation», XXXIII, n.1, pp. 9-10.

Cox, G.W. (1997), Making Votes Count. Strategic Coordination in the World's Electoral Systems, Cambridge, Cambridge University Press.

Craig, A.L. e W.A. Cornelius (1995), Houses Divided: Parties and Political Reform in Mexico, in S. Mainwaring e T.R. Scully (a cura di), Building Democratic Institutions. Party Systems in Latin America, Stanford, Stanford University Press, pp. 249-257.

Crampton, R.J. (1991), The Bulgarian Elections of 1990, in «Representation», XXIX, n. 108, pp. 33-35.

D'Alimonte, R. e A. Chiaramonte (1993), Il nuovo sistema elettorale italiano: quali opportunità?, in «Rivista Italiana di Scienza Politica», XXIII, pp. 513-547.

Dunleavy, P. e H. Margetts (1995), Understanding the Dynamics of Electoral Reform, in «International Political Science Review», XVI, pp. 9-29.

Duverger, M. (1951), Les partis politiques, Paris, Colin; trad. it. I partiti politici, Milano, Comunità, 1970.

Elklit, J. (1992), The Best of Both Worlds? The Danish Electoral System 1915-1920 in a Comparative Perspective, in «Electoral Studies», XI, pp. 189-205.

Fitzmaurice, J. (1995), The Hungarian Elections of May 1994, in «Electoral Studies», XIV, pp. 77-80. 
Foeweraker, J. e T. Landman (1995), The August 1994 Elections in Mexico, in «Electoral Studies», vol. 14, pp. 199-203.

Gabel, M.J. (1995), The Political Consequences of Electoral Laws in the 1990 Hungarian Elections, in «Comparative Politics», XXVII, pp. 205-214.

Hsieh, J.F. e E. Niou (1996), Taiwan's March 1996 Elections, in «Electoral Studies», XV, pp. 545-550.

International Foundation for Electoral Systems (1995), Comparison of Russian Election Laws, Moscow.

Inter-Parliamentary Union, Chronicle of Parliamentary Elections and Development, Gineva, anni vari.

Irvine, W.P. (1984), 'Additional-Member' Electoral Systems, in Lijphart e Grofman (1984), pp. 165-174.

Jeffery, C. (1995), The German Bundestag Election of 1994, in «Representation», XXXI, n. 1, pp. 13-16.

Jesse, E. (1988), Split-Voting in The Federal Republic of Germany: An Analysis of The Federal Elections from 1953 to 1987, in «Electoral Studies», VII, pp. 109-124.

- (1995), The Method of Electing the German Bundestag and its Effect on the Parties, in «Representation», XXXIII, n. 1, pp. 5-8.

Jones, M.P. (1995), A Guide to the Electoral Systems of the Americas, in «Electoral Studies», XIV, pp. 5-21.

Kaase, M. (1984), Personalized Proportional Representation: The 'Model' of the West German Electoral System, in Lijphart e Grofman (1984), pp. 155-164.

Kasapovic, M. (1995), Segmented or «Entrenched» Electoral Systems, in «Politicka Misao-Croatian Political Science Review», XXXII, pp. 173-186.

- (1996), 1995 Parliamentary Elections in Croatia, in «Electoral Studies», XV, pp. 269-282.

Koulov, B. (1995), Geography of Electoral Preferences: The 1990 Great National Assembly Elections in Bulgaria, in «Political Geography», XIV, pp. 241-258.

Kukorelli, I. (1991), The Birth, Testing and Results of the 1989 Hungarian Electoral Law, in «Soviet Studies», XLIII, pp. 137-156.

Laasko, M. e R. Taagepera (1979), «Effective» Number of Parties: A Measure with Application to West Europe, in «Comparative Political Studies», XII, pp. 3-27.

Lakeman, E. (1970), How Democracies Vote: A Study of Electoral Systems, London, Faber \& Faber.

Landman, T. (1995), 'El Chiripero' Wins: The Venezuelan Elections of 1993, in «Electoral Studies», XIV, pp. 100-104.

Lijphart, A. (1984), Trying to Have the Best of Both Worlds: Semi-Proportional and Mixed Systems, in Lijphart e Grofman (1984), pp. 207-213.

- (1986), Proportionality by Non-PR Methods: Etbnic Representation 
in Belgium, Cyprus, Lebanon, New Zealand, West Germany and Zimbabwe, in B. Grofman e A. Lijphart (a cura di), Electoral Laws and Their Political Consequences, New York, Agathon, pp. 113-123.

- (1987), The Demise of The Last Westminster System? Comments on The Report of New Zealand's Royal Commission on The Electoral System, in «Electoral Studies», VI, pp. 97-103.

- (1994), Electoral Systems and Party Systems. A Study of Twenty-Seven Democracies, 1945-1990, Oxford, Oxford University Press.

Lijphart, A. e B. Grofman (a cura di) (1984), Choosing an Electoral System: Issues and Alternatives, New York, Praeger.

Loosemore, J. e V.J. Hanby (1971), The Theoretical Limits of Maximum Distorsion: Some Analytic Expression for Electoral Systems, in «British Journal of Political Science», I, pp. 467-477.

Mackerras, M. (1994), Reform of New Zealand's Voting System, 19851996, in «Representation», XXXII, n. 118, pp. 36-40.

Martis, K.C., Z. Kovacs, D. Kovacs e S. Peter (1992), The Geography of the 1990 Hungarian Parliamentary Elections, in «Political Geography», XI, pp. 283-305.

Monroe, B.L. (1994), Understanding Electoral Systems: Beyond Plurality versus $P R$, in «Political Science \& Politics», XXVII, pp. 677682.

Morriss, P. (1996), Electoral Politics in South Korea, in «Electoral Studies», XV, pp. 550-562.

Moser, R.G. (1995), The Impact of the Electoral System on Post-Communist Party Development: The Case of the 1993 Russian Parliamentary Elections, in «Electoral Studies», XIV, pp. 377-398.

Nagel, J.H. (1994), How Many Parties Will New Zealand Have under MMP?, in «Political Science», IVL, pp. 139-160.

Nohlen, D. (1978), Wablsysteme der Welt. Daten und Analysen. Ein Handbuch, München, Piper.

- (1984a), Two Incompatible Principles of Representation, in Lijphart e Grofman (1984), pp. 83-89.

- (1984b), Changes and Choices in Electoral Systems, in Lijphart e Grofman (1984), pp. 217-224.

Racz, B. (1991), Political Pluralisation in Hungary: The 1990 Elections, in «Soviet Studies», ILIII, pp. 107-136.

Rady, M. (1994), The 1994 Hungarian General Election, in «Representation», XXXII, n. 119, pp. 69-72.

Rae, D.W. (1967), The Political Consequences of Electoral Laws, New Haven, Yale University Press.

Reeve, A. e Ware A.J. (1992), Electoral Systems: A Comparative and Theoretical Introduction, London, Routledge.

Rokkan, S. (1970) Citizens, Elections, Parties, Oslo, Universitetsforlaget; trad. it. Cittadini, elezioni, partiti, Bologna, Il Mulino, 1982. 
Rose, R. (1984), Electoral Systems: A Question of Degree or of Principle?, in Lijphart e Grofman (1984), pp. 73-81.

Sartori, G. (1968), Representational Systems, in International Enciclopaedia of the Social Sciences, New York, Macmillan-The Free Press, XIII, pp. 465-474.

- (1984), Le «leggi» sulla influenza dei sistemi elettorali, in «Rivista Italiana di Scienza Politica», XIV.

Shiratori, R. (1995), The Politics of Electoral Reform in Japan, in «International Political Science Review», XVI, pp. 79-94.

Shugart, M.S. (1985), The Two Effects of District Magnitude: Venezuela as a Crucial Experiment, in «European Journal of political Research», XIII, pp. 353-364.

Szajkowski, B. (1994), Albania, in AA.VV., Political Parties of Eastern Europe, Russia and the Successor States, Harlow, Longman, pp. 128.

The Norwegian Helsinki Committee (1995), Report from the Observation of the Parliamentary and Presidential Elections of the Republic of Georgia, 5 November 1995.

- (1996a), Report from the Parliamentary Elections in Albania, 26 May 1996.

- (1996b), Report from the Observation of the Parliamentary Elections in the Russian Federation 17 December 1995.

Taagepera, R. e M.S. Shugart (1989), Seats \& Votes. The Effects and Determinants of Electoral Systems, New Haven-London, Yale University Press.

Vowles, J. (1995), The Politics of Electoral Reform in New Zealand, in «International Political Science Review», XVI, pp. 95-115.

Wyman, M., B. Miller, S. White e P. Heywood (1994), The Russian Elections of December 1993, in «Electoral Studies», XIII, pp. 254271. 